\title{
A LOW-MEMORY APPROACH FOR BEST-STATE ESTIMATION OF HIDDEN MARKOV MODELS WITH MODEL ERROR*
}

\author{
MIHAI ANITESCU ${ }^{\dagger}$, XIAOYAN ZENG ${ }^{\ddagger}$, AND EMIL M. CONSTANTINESCU ${ }^{\dagger}$
}

\begin{abstract}
We present a low-memory approach for the best-state estimate (data assimilation) of hidden Markov models where model error is considered. In particular, our findings apply to the 4DVar framework. The novelty of our approach resides in the fact that the storage needed by our estimation framework, while including model error, is dramatically reduced from $\mathcal{O}$ (number of time steps) to $\mathcal{O}(1)$. The main insight is that we can restate the objective function of the state estimation (the likelihood function) from a function of all states to a function of the initial state only. We do so by restricting the other states by recursively enforcing the optimality conditions. This results in a regular nonlinear equation or an optimization problem for which a descent direction can be computed using only a forward sweep. In turn, the best estimate can be obtained locally by limited-memory quasi-Newton algorithms that need only $\mathcal{O}(1)$ storage with respect to the time steps. Our findings are demonstrated by numerical experiments on Burgers' equations.
\end{abstract}

Key words. data assimilation, weakly constrained 4D-Var, hidden Markov models, limitedmemory methods, quasi-Newton methods

AMS subject classifications. 90C53, 93E10, 62M05

DOI. $10.1137 / 120870451$

1. Introduction. Data assimilation is the process of computing the best estimate of the trajectory of a dynamical system with observational data $[6,9,10]$. This technique is used extensively in meteorology and hydrology in order to make accurate predictions about the state of the atmosphere and oceans $[9,14]$. However, recent applications have called for explicit inclusion of model error such as from subgrid modeling, boundary conditions, and forcings. All these modeling uncertainties are aggregated into a component that is generically called model error $[8,16,18]$, which in turn results in the following best-fit 4D-Var-with-model-error functional $[12,13,24,25,28,26]$ :

$$
\begin{aligned}
\mathcal{J}\left(x_{t_{0}}, x_{t_{1}}, \ldots, x_{t_{N}}\right)= & \frac{1}{2}\left(x_{t_{0}}-x_{B}\right)^{T} Q_{B}^{-1}\left(x_{t_{0}}-x_{B}\right) \\
& +\frac{1}{2} \sum_{k=0}^{N}\left(\mathcal{H}_{k}\left(x_{t_{k}}\right)-y_{k}\right)^{T} R_{k}^{-1}\left(\mathcal{H}_{k}\left(x_{t_{k}}\right)-y_{k}\right) \\
& +\frac{1}{2} \sum_{k=0}^{N-1}\left(x_{t_{k+1}}-\mathcal{M}_{k}\left(x_{t_{k}}\right)\right)^{T} Q_{k}^{-1}\left(x_{t_{k+1}}-\mathcal{M}_{k}\left(x_{t_{k}}\right)\right) .
\end{aligned}
$$

${ }^{*}$ Received by the editors March 19, 2012; accepted for publication (in revised form) October 10, 2013; published electronically February 18, 2014. This work was supported by the Department of Energy under contract DE-AC02-06CH11357. The U.S. Government retains a nonexclusive, royaltyfree license to publish or reproduce the published form of this contribution, or allow others to do so, for U.S. Government purposes. Copyright is owned by SIAM to the extent not limited by these rights.

http://www.siam.org/journals/sinum/52-1/87045.html

${ }^{\dagger}$ Mathematics and Computer Science Division, Argonne National Laboratory, Argonne, IL 60439 (anitescu@mcs.anl.gov, emconsta@mcs.anl.gov).

${ }^{\ddagger}$ Corresponding author. Mathematics Department, Shanghai University, Baoshan, Shanghai, People's Republic of China (cherryzxy@shu.edu.cn), and Mathematics and Computer Science Division, Argonne National Laboratory, Argonne, IL 60439 (zeng@mcs.anl.gov). 
All the quantities of interest are indexed by $k, k=0,1, \ldots, N$, where $t_{k}$ is the time instant. Here, the variables $x_{t_{k}}$ are the states of the model at times $t_{i}$ that need to be identified by minimizing the functional $\mathcal{J}$. The data of the problem are as follows. The quantities $x_{B}$ and $Q_{B}$ are the background state and the background covariance matrix, respectively. The vectors $y_{k}$ represent the observations, whereas the nonlinear mapping $\mathcal{H}(\cdot)$ is the observation operator that maps states into observables. The matrix $R_{k}$ is the covariance error for the observations. The mapping $M_{k}(\cdot)$ describes the evolution of the physical model, whereas the matrix $Q_{k}$ quantifies the covariance of the model error. The functional $\mathcal{J}$ is the minus log likelihood of the hidden Markov model $[21,22]$ :

$$
x_{t_{k+1}}=M\left(x_{t_{k}}\right)+\eta_{k}, \quad y_{k}=\mathcal{H}\left(x_{t_{k}}\right)+\varepsilon_{k}, \quad \eta_{k} \sim \mathcal{N}\left(\mathbf{0}, Q_{k}\right) \varepsilon_{k} \sim \mathcal{N}\left(\mathbf{0}, R_{k}\right) .
$$

For this reason, we call the minimization of $\mathcal{J}$, which is equivalent to the maximum likelihood calculation for the hidden Markov model, the state estimation of hidden Markov models with model error.

In the limiting case of 0 model error, that is, $Q_{k}^{-1} \rightarrow \infty$, we obtain the so-called strongly constrained model $[5,9,20]$, which is the one most commonly used in today's applications. Because it now includes the recursive constraints $x_{t_{k+1}}=M_{k}\left(x_{t_{k}}\right)$, it can effectively be thought of as a function only of the initial condition $x_{t_{0}}$, which is the only variable that needs to be stored, with all the others being obtained by the recursion.

Unfortunately, this reduction does not apply to the case including model error, also called weakly constrained, which is now a function of $N+1$ times more variables and thus requires substantially more memory to store the result of the minimization of (1.1). As we move to even higher spatial resolution such as global cloud resolving models that require a horizontal resolution of $1-3 \mathrm{Km}^{2}$, the amount of memory and storage space in the case of considering model error would make such computations out of practical reach. We focus on memory requirements because we are entering a phase in computational science where power considerations lead us to reduced available memory per unit of computational power (see [7]).

In this study we introduce a numerical method that reduces the memory requirements of running the weakly constrained $4 \mathrm{D}$-Var. The method is based on a shooting philosophy constrained by the optimality conditions for the likelihood function. Burgers' equation is used to illustrate the technique and compare it with a derivative-free or full memory-intensive implementation. While this will be done in a 1+1D-Var (in the sense that the spatial dimension is only 1), our example has the same time-dependence structure as full 4D-Var approaches. Therefore, we expect that conclusions about the dependence of the storage requirements of the method on the number of time stepsthe main investigation topic here - will carry through to the actual 4D-Var case.

The rest of the paper is structured as follows. In section 2 we present our algorithm in an abstract framework, and we analyze its well-posedness. In section 3 we discuss the stability and conditions and considerations for our low-memory algorithms. Numerical experiments to validate our findings are presented in section 5 . In section 6 we summarize our conclusions.

2. A low-memory approach for data assimilation with model error. We introduce an abstraction of the data assimilation with the model error problem, the 4D-Var problem (1.1). The abstraction will be useful in understanding the fundamentals of our approach, reducing the notation burden, and providing a framework for the extension of these results. 
2.1. Abstraction of the problem. We assume that we are trying to recover the states $\boldsymbol{x}_{i}, i=0,1, \ldots, N$, of a system that evolves over $N$ time steps with $\boldsymbol{x}_{0}$ as an initial state and $\boldsymbol{x}_{N}$ as a final state.

We assume that this optimal state is recovered by minimizing a cost functional with several components. These components are of the following two types:

- Evolution components, which constrain the relative evolution of two consecutive components, $\phi_{i}\left(\boldsymbol{x}_{i}, \boldsymbol{x}_{i+1}\right), i=0,1, \ldots, N-1$.

- Observational components, which constrain each state either by means of observations or by means of a background prior, $\gamma_{i}, i=0,1, \ldots, N$.

We define the scaled cost functional $\Gamma$ as

$$
\Gamma\left(\boldsymbol{x}_{0: N}\right):=\frac{1}{N}\left(\sum_{i=0}^{N-1}\left[\gamma_{i}\left(\boldsymbol{x}_{i}\right)+\phi_{i}\left(\boldsymbol{x}_{i}, \boldsymbol{x}_{i+1}\right)\right]+\gamma_{N}\left(\boldsymbol{x}_{N}\right)\right) .
$$

Minimizing this functional $\Gamma$ will result in the best estimate according to the $\Gamma$ criterion. The rescaling will not affect the solution of the problem, but it is useful in comparing residuals for increasing $N$. We will ignore the rescaling in the theoretical derivations, but we will use it when comparing the numerical results.

A key element in proving our results is the following assumption.

Assumption 1. Assume that $\phi_{i}\left(\boldsymbol{x}_{i}, \boldsymbol{x}_{i+1}\right)$ and $\gamma_{i}\left(\boldsymbol{x}_{i}\right)$ are twice continuously differentiable and that the mixed differentiation function $\nabla_{\boldsymbol{x}_{i+1} \boldsymbol{x}_{i}}^{2} \phi_{i}\left(\boldsymbol{x}_{i}, \boldsymbol{x}_{i+1}\right)$ is invertible in the neighborhood of the minimum $x_{0: N}^{*}$.

2.2. Illustration of the abstraction in the case of $4 \mathrm{D}$-Var. In the case of the $4 \mathrm{D}$-Var approach (1.1), we have that, for $i=0, \ldots, N-1$,

$$
\phi_{i}\left(\boldsymbol{x}_{i}, \boldsymbol{x}_{i+1}\right)=\frac{1}{2}\left(\boldsymbol{x}_{i+1}-\mathcal{M}_{i}\left(\boldsymbol{x}_{i}\right)\right)^{T} Q_{i}^{-1}\left(\boldsymbol{x}_{i+1}-\mathcal{M}_{i}\left(\boldsymbol{x}_{i}\right)\right)
$$

corresponds to the model error. Also, for $i=1, \ldots, N$,

$$
\gamma_{i}\left(\boldsymbol{x}_{i}\right)=\frac{1}{2}\left(\boldsymbol{y}_{i}-\mathcal{H}_{i}\left(\boldsymbol{x}_{i}\right)\right)^{T} R_{i}^{-1}\left(\boldsymbol{y}_{i}-\mathcal{H}_{i}\left(\boldsymbol{x}_{i}\right)\right)
$$

corresponds to the difference between observations and its model counterparts. For $i=0, \gamma_{0}$ includes the background error measurement for the current value of $\boldsymbol{x}_{0}$ and is formulated as

$$
\gamma_{0}\left(\boldsymbol{x}_{0}\right)=\frac{1}{2}\left(\boldsymbol{x}_{0}-\boldsymbol{x}_{B}\right)^{T} Q_{B}^{-1}\left(\boldsymbol{x}_{0}-\boldsymbol{x}_{B}\right)+\frac{1}{2}\left(\boldsymbol{y}_{0}-\mathcal{H}_{0}\left(\boldsymbol{x}_{0}\right)\right)^{T} R_{0}^{-1}\left(\boldsymbol{y}_{0}-\mathcal{H}_{0}\left(\boldsymbol{x}_{0}\right)\right) .
$$

Here $\boldsymbol{x}_{i}=x_{t_{i}}$ denotes a state in the $i$ th step. We use $\boldsymbol{x}_{0: N}$ to represent $\left[\boldsymbol{x}_{0}, \ldots, \boldsymbol{x}_{N}\right]^{T}$ for shorthand.

Concerning Assumption 1, we note that for the weakly constrained 4D-Var approach defined in $(2.1),(2.2),(2.3)$, and (2.4) we know that

$$
\nabla_{\boldsymbol{x}_{i+1}} \nabla_{\boldsymbol{x}_{i}} \phi_{i}\left(\boldsymbol{x}_{i}, \boldsymbol{x}_{i+1}\right)=-\left(\nabla_{\boldsymbol{x}_{i}} \mathcal{M}_{i}\left(\boldsymbol{x}_{i}\right)\right)^{T} Q^{-1} .
$$

Therefore the matrix on the left is invertible if and only if $\nabla_{\boldsymbol{x}_{i}} \mathcal{M}_{i}\left(\boldsymbol{x}_{i}\right)$ is invertible. In addition, for satisfying Assumption 1 completely, $\mathcal{M}_{i}, \mathcal{H}_{i}$ must be continuously differentiable.

Since in most applications $\mathcal{M}_{i}$ represents the solution flow of a regular ordinary differential equation, the assumption that $\mathcal{M}_{i}$ is smooth and invertible holds. Since the observation operator $\mathcal{H}_{i}$ can indeed be assumed to be continuously differentiable, we conclude that Assumption 1 holds in this case. 
2.3. Reduced-memory algorithm. In this section, our goal is to define an algorithm to minimize functional $\Gamma$ as in (2.1), while storing at any time only a small number of $\left\{\boldsymbol{x}_{i}\right\}$.

We define a sequence of functions as follows:

$$
\begin{aligned}
& \theta_{0}\left(\boldsymbol{x}_{0}, \boldsymbol{x}_{1}\right):=\nabla_{\boldsymbol{x}_{0}} \phi_{0}+\nabla_{\boldsymbol{x}_{0}} \gamma_{0} \\
& \theta_{i}\left(\boldsymbol{x}_{i-1}, \boldsymbol{x}_{i}, \boldsymbol{x}_{i+1}\right):=\nabla_{\boldsymbol{x}_{i}} \phi_{i}+\nabla_{\boldsymbol{x}_{i}} \phi_{i-1}+\nabla_{\boldsymbol{x}_{i}} \gamma_{i}, \quad i=1, \ldots, N-1, \\
& \theta_{N}\left(\boldsymbol{x}_{N-1}, \boldsymbol{x}_{N}\right):=\nabla_{\boldsymbol{x}_{N}} \phi_{N-1}+\nabla_{\boldsymbol{x}_{N}} \gamma_{N} .
\end{aligned}
$$

It immediately follows from (2.1) that the following relationships hold for the partial derivatives of $\Gamma$ :

$$
\begin{aligned}
& \nabla_{\boldsymbol{x}_{0}} \Gamma\left(x_{0: N}\right)=\frac{1}{N} \theta_{0}\left(\boldsymbol{x}_{0}, \boldsymbol{x}_{1}\right), \\
& \nabla_{\boldsymbol{x}_{i}} \Gamma\left(x_{0: N}\right)=\frac{1}{N} \theta_{i}\left(\boldsymbol{x}_{i-1}, \boldsymbol{x}_{i}, \boldsymbol{x}_{i+1}\right), \quad i=1, \ldots, N-1, \\
& \nabla_{\boldsymbol{x}_{N}} \Gamma\left(x_{0: N}\right)=\frac{1}{N} \theta_{N}\left(\boldsymbol{x}_{N-1}, \boldsymbol{x}_{N}\right) .
\end{aligned}
$$

The core of our method is based on the following observation.

THEOREM 1. Under Assumption 1, there exist continuously differentiable mappings $\lambda_{i}\left(\boldsymbol{x}_{0}\right), i=1,2, \ldots, N$, such that

$$
\begin{aligned}
& \theta_{0}\left(\boldsymbol{x}_{0}, \lambda_{1}\left(\boldsymbol{x}_{0}\right)\right)=0, \\
& \theta_{i}\left(\lambda_{i-1}\left(\boldsymbol{x}_{0}\right), \lambda_{i}\left(\boldsymbol{x}_{0}\right), \lambda_{i+1}\left(\boldsymbol{x}_{0}\right)\right)=0, \quad i=1,2, \ldots, N-1 .
\end{aligned}
$$

Moreover, for any $\boldsymbol{x}_{0},\left\{\lambda_{i}\left(\boldsymbol{x}_{0}\right)\right\}_{i=1,2 \ldots, N}$ are the unique vectors with this property.

Proof. We have from the definition of $\theta_{i}$ (while temporarily dropping the obvious dependence on $\boldsymbol{x}_{0}$ ) that

$$
\nabla_{\boldsymbol{x}_{i+1}} \theta_{i}\left(\boldsymbol{x}_{i-1}, \boldsymbol{x}_{i}, \boldsymbol{x}_{i+1}\right)=\nabla_{\boldsymbol{x}_{i+1}} \nabla_{\boldsymbol{x}_{i}} \phi_{i}\left(\lambda_{i}, \lambda_{i+1}\right) .
$$

From Assumption 1 we have that $\nabla_{\boldsymbol{x}_{i+1} \boldsymbol{x}_{i}}^{2} \phi_{i}\left(\lambda_{i}, \lambda_{i+1}\right)$ is invertible in the neighborhood of $x_{0 . N}^{*}$, which in turn makes the Jacobian of the associated nonlinear equation in (2.9) invertible in $\boldsymbol{x}_{i+1}, i=1,2, \ldots, N-1$ (with a similar conclusion for $i=0$ ). The conclusion follows from application of the implicit function theorem recursively in $(2.9)$.

In the case of the 4D-Var functional (1.1), the mappings $\lambda_{i}\left(x_{0}\right)$ can explicitly be computed as follows. Because of the quasi-quadratic form of $\phi_{i}(2.2)$ and $\gamma_{i}(2.3)$, for a fixed initial state $\boldsymbol{x}_{0}$, we get $\boldsymbol{x}_{1}$ by solving the $\theta_{0}\left(\boldsymbol{x}_{0}, \boldsymbol{x}_{1}\right)=0$ as

$$
\begin{aligned}
\boldsymbol{x}_{1}= & \mathcal{M}_{0}\left(\boldsymbol{x}_{0}\right)+Q_{0}\left(\nabla_{\boldsymbol{x}_{0}} \mathcal{M}_{0}\left(\boldsymbol{x}_{0}\right)\right)^{-T} Q_{B}^{-1}\left(\boldsymbol{x}_{0}-\boldsymbol{x}_{B}\right) \\
& +Q_{0}\left(\nabla_{\boldsymbol{x}_{0}} \mathcal{M}_{0}\left(\boldsymbol{x}_{0}\right)\right)^{-T}\left(\nabla_{\boldsymbol{x}_{0}} \mathcal{H}_{0}\left(\boldsymbol{x}_{0}\right)\right)^{T} R_{0}^{-1}\left(\mathcal{H}_{0}\left(\boldsymbol{x}_{0}\right)-\boldsymbol{y}_{0}\right),
\end{aligned}
$$

and we get $\boldsymbol{x}_{i+1}$ by solving $\theta_{i}\left(\boldsymbol{x}_{i-1}, \boldsymbol{x}_{i}, \boldsymbol{x}_{i+1}\right)=0$ for $i=1, \ldots, N-1$ as

$$
\begin{aligned}
\boldsymbol{x}_{i+1}= & \mathcal{M}_{i}\left(\boldsymbol{x}_{i}\right)+Q_{i}\left(\nabla_{\boldsymbol{x}_{i}} \mathcal{M}_{i}\left(\boldsymbol{x}_{i}\right)\right)^{-T}\left(\nabla_{\boldsymbol{x}_{i}} \mathcal{H}_{i}\left(\boldsymbol{x}_{i}\right)\right)^{T} R_{i}^{-1}\left(\mathcal{H}_{i}\left(\boldsymbol{x}_{i}\right)-\boldsymbol{y}_{i}\right) \\
& +Q_{i}\left(\nabla_{\boldsymbol{x}_{i}} \mathcal{M}_{i}\left(\boldsymbol{x}_{i}\right)\right)^{-T} Q_{i-1}^{-1}\left(\boldsymbol{x}_{i}-\mathcal{M}_{i-1}\left(\boldsymbol{x}_{i-1}\right)\right) .
\end{aligned}
$$

Based on Theorem 1, we can rewrite $\Gamma$ as a function of $\boldsymbol{x}_{0}$ as follows:

$$
\widehat{\Gamma}\left(\boldsymbol{x}_{0}\right)=\frac{1}{N}\left[\sum_{i=0}^{N-1} \gamma_{i}\left(\lambda_{i}\left(\boldsymbol{x}_{0}\right)\right)+\phi_{i}\left(\lambda_{i}\left(\boldsymbol{x}_{0}\right), \lambda_{i+1}\left(\boldsymbol{x}_{0}\right)\right)+\gamma_{N}\left(\lambda_{N}\left(\boldsymbol{x}_{0}\right)\right)\right],
$$


with $\lambda_{0}\left(\boldsymbol{x}_{0}\right)=\boldsymbol{x}_{0}$. By transferring the cost function (2.1) into (2.12), a function of initial state, considerable storage space is saved during computation since we reduce the multistate function to a single-state function. The main vehicle for this reduction is the explicit enforcement of the optimality conditions at each of the time steps other than the initial one; however, these lead to a local minimizer only when $\boldsymbol{x}_{0}$ is the same as the first component of the minimizer. In some sense, the optimality conditions become the strong constraint in the approach, replacing the perfect model assumption from current 4D-Var data assimilation procedures, that is, of course, if we can manipulate the function $\widehat{\Gamma}$ as required by the optimization algorithms in a way that maintains an $\mathcal{O}(1)$ storage.

To that end, we need more theoretical support to verify that the optimum solution of (2.12) is the same as the initial state of the original problem's optimum solution. It is well known that for a twice continuously differentiable function $f$, if $x$ is a local minimizer of $f$, then the following two necessary conditions must be satisfied: $f^{\prime}(x)$ equals 0 (first-order necessary condition; $x$ here is called a stationary point) and $f^{\prime \prime}(x)$ is positive semidefinite (second-order necessary condition). The sufficient conditions needed for $x$ to be a local minimizer of $f$ are that $x$ is a stationary point and $f^{\prime \prime}(x)$ is positive definite (second-order sufficient condition). Hence we need to figure out the derivatives first.

The gradient of $\widehat{\Gamma}$ is calculated as

$$
\nabla_{\boldsymbol{x}_{0}} \widehat{\Gamma}=\theta_{0}\left(\lambda_{0}, \lambda_{1}\right)+\left(\nabla_{\boldsymbol{x}_{0}} \lambda_{N}\right)^{T} \theta_{N}\left(\lambda_{N-1}, \lambda_{N}\right)+\sum_{i=1}^{N-1}\left(\nabla_{\boldsymbol{x}_{0}} \lambda_{i}\right)^{T} \theta_{i}\left(\lambda_{i-1}, \lambda_{i}, \lambda_{i+1}\right) .
$$

Because of the way $\lambda_{i}, i=1, \ldots, N$, are computed from the recursion (2.9), which implies that $\theta_{0}\left(\lambda_{0}, \lambda_{1}\right) \equiv 0$ and $\theta_{i}\left(\lambda_{i-1}, \lambda_{i}, \lambda_{i+1}\right) \equiv 0, \quad i=1, \ldots, N-1$, we have that, in the neighborhood of $\boldsymbol{x}_{0}^{*}$,

$$
\nabla_{\boldsymbol{x}_{0}} \widehat{\Gamma}=\left(\nabla_{\boldsymbol{x}_{0}} \lambda_{N}\right)^{T} \theta_{N}\left(\lambda_{N-1}, \lambda_{N}\right) .
$$

Define $L_{i}:=\nabla_{\boldsymbol{x}_{0}} \lambda_{i}$. The second-order derivative of $\widehat{\Gamma}$ at $\boldsymbol{x}_{0}^{*}$ is calculated by product rule as

$$
\begin{aligned}
\nabla_{\boldsymbol{x}_{0} \boldsymbol{x}_{0}}^{2} & \widehat{\Gamma}=\nabla_{\lambda_{0}} \theta_{0}+L_{1}^{T} \nabla_{\lambda_{1}} \theta_{0}+\left(L_{N-1}^{T} \nabla_{\lambda_{N-1}} \theta_{N}+L_{N}^{T} \nabla_{\lambda_{N}} \theta_{N}\right) L_{N} \\
+ & \sum_{i=1}^{N-1}\left(L_{i-1}^{T} \nabla_{\lambda_{i-1}} \theta_{i}+L_{i}^{T} \nabla_{\lambda_{i}} \theta_{i}+L_{i+1}^{T} \nabla_{\lambda_{i+1}} \theta_{i}\right) L_{i} \\
+ & \sum_{i=1}^{N-1}\left(\left(\theta_{i}\right)^{T} \otimes I_{s}\right) \nabla_{\boldsymbol{x}_{0}} \operatorname{vec}\left(L_{i}\right)+\left(\left(\theta_{N}\right)^{T} \otimes I_{s}\right) \nabla_{\boldsymbol{x}_{0}} \operatorname{vec}\left(L_{N}\right),
\end{aligned}
$$

where $I_{s}$ is an $s \times s$ identity matrix with $s$ being the dimension of $\boldsymbol{x}_{i}$ and $\otimes$ denotes Kronecker product. To prove (2.14), we need only prove that the first derivative matrix of $s \times s$ matrix $M$ and $s \times 1$ vector $\boldsymbol{u}$, with respect to $s \times 1$ vector $\boldsymbol{x}$, i.e.,

$$
\nabla_{\boldsymbol{x}}(M \boldsymbol{u})=\left(\boldsymbol{u}^{T} \otimes I_{s}\right) \nabla_{\boldsymbol{x}} \operatorname{vec}(M)+M \nabla_{\boldsymbol{x}} \boldsymbol{u} .
$$

Here the Kronecker product is $\boldsymbol{u}^{T} \otimes I_{s}=\left(\begin{array}{lll}u_{1} I_{s} & \cdots & u_{s} I_{s}\end{array}\right)$, and $\operatorname{vec}(M)$ is an $s^{2} \times 1$ vector stacking the columns of the matrix $M$ on top of one another; that is, $\operatorname{vec}(M)=\left(\begin{array}{lllllll}m_{11} & \cdots & m_{s, 1} & \cdots & m_{1 s} & \cdots & m_{s, s}\end{array}\right)^{T}$. The first derivative matrix 
of $\operatorname{vec}(M)$ is

$$
\nabla_{\boldsymbol{x}} \operatorname{vec}(M)=\left(\begin{array}{ccc}
\frac{\partial m_{11}}{\partial x_{1}} & \cdots & \frac{\partial m_{11}}{\partial x_{s}} \\
\vdots & \vdots & \vdots \\
\frac{\partial m_{s s}}{\partial x_{1}} & \cdots & \frac{\partial m_{s s}}{\partial x_{s}}
\end{array}\right)
$$

Hence the $i$ th-row-and- $j$ th-column element of $\left(\boldsymbol{u}^{T} \otimes I_{s}\right) \nabla_{\boldsymbol{x}} \operatorname{vec}(M)$ is $\sum_{k=1}^{s} \frac{\partial m_{i k}}{\partial x_{j}} u_{k}$. The $i$ th-row-and- $j$ th-column element of $M \nabla_{\boldsymbol{x}} \boldsymbol{u}$ is $\sum_{k=1}^{s} m_{i, k} \frac{\partial u_{k}}{\partial x_{j}}$. The $i$ th-row element of $\boldsymbol{M} \boldsymbol{u}=\sum_{k=1}^{s} m_{i k} u_{k}$, and hence the $i$ th-row-and- $j$ th-column element of $\nabla_{\boldsymbol{x}}(M \boldsymbol{u})$, is $\sum_{k=1}^{s} m_{i k} \frac{\partial u_{k}}{\partial x_{j}}+\sum_{k=1}^{s} \frac{\partial m_{i k}}{\partial x_{j}} u_{k}$. Hence (2.14) is verified.

From Theorem 1, the last line of (2.14) is zero. Then (2.14) can be simplified at $x_{0}^{*}$ to

$$
\begin{aligned}
\nabla_{\boldsymbol{x}_{0} \boldsymbol{x}_{0}}^{2} \widehat{\Gamma}= & \nabla_{\lambda_{0}} \theta_{0}+L_{1}^{T} \nabla_{\lambda_{1}} \theta_{0}+\left(L_{N-1}^{T} \nabla_{\lambda_{N-1}} \theta_{N}+L_{N}^{T} \nabla_{\lambda_{N}} \theta_{N}\right) L_{N} \\
& +\sum_{i=1}^{N-1}\left(L_{i-1}^{T} \nabla_{\lambda_{i-1}} \theta_{i}+L_{i}^{T} \nabla_{\lambda_{i}} \theta_{i}+L_{i+1}^{T} \nabla_{\lambda_{i+1}} \theta_{i}\right) L_{i}^{T} .
\end{aligned}
$$

Because $\nabla_{\lambda_{j}} \theta_{i}=\left.\nabla_{\boldsymbol{x}_{j}} \nabla_{\boldsymbol{x}_{i}} \Gamma\right|_{\boldsymbol{x}_{j}=\lambda_{j}}, j=i-1, i, i+1$, one can easily verify that

$$
\nabla_{\boldsymbol{x}_{0} \boldsymbol{x}_{0}}^{2} \widehat{\Gamma}=\Lambda^{T}\left(\nabla_{\boldsymbol{x}_{0: N}}^{2} \Gamma\left(\boldsymbol{x}_{0}, \lambda_{1: N}\right)\right) \Lambda
$$

where

$$
\Lambda^{T}=\left[I,\left(\nabla_{\boldsymbol{x}_{0}} \lambda_{1}\right)^{T}, \ldots,\left(\nabla_{\boldsymbol{x}_{0}} \lambda_{N}\right)^{T}\right] .
$$

From (2.6) and (2.13), as well as the definition of the mappings $\theta_{i}$, it immediately follows that the component $\boldsymbol{x}_{0}^{*}$ of a stationary point $\boldsymbol{x}_{0}^{*}, \boldsymbol{x}_{1}^{*}, \ldots, \boldsymbol{x}_{N}^{*}$ of (2.1) also satisfies $\nabla_{\boldsymbol{x}_{0}} \widehat{\Gamma}\left(\boldsymbol{x}_{0}^{*}\right)=0$. Therefore, it is a stationary point of $\widehat{\Gamma}$. In the following result, we show that the reciprocal is also true under some mild assumptions.

THEOREM 2. If $\boldsymbol{x}_{0}^{*}$ is a local minimizer of $\widehat{\Gamma}\left(\boldsymbol{x}_{0}\right)$ and $\nabla_{\boldsymbol{x}_{0}} \lambda_{N}\left(\boldsymbol{x}_{0}\right)$ is invertible, then $\left(\boldsymbol{x}_{0}^{*}, \lambda_{1}\left(\boldsymbol{x}_{0}^{*}\right), \ldots, \lambda_{N}\left(\boldsymbol{x}_{0}^{*}\right)\right)$ is a stationary point of $\Gamma\left(\boldsymbol{x}_{0: N}\right)$.

Proof. From the definition of the mapping $\lambda_{i}(\cdot)$ in Theorem 1, we have that for $i=1, \ldots, N-1$,

$$
\theta_{0}\left(\boldsymbol{x}_{0}^{*}, \lambda_{1}\left(\boldsymbol{x}_{0}^{*}\right)\right)=0, \theta_{i}\left(\lambda_{i-1}\left(\boldsymbol{x}_{0}^{*}\right), \lambda_{i}\left(\boldsymbol{x}_{0}^{*}\right), \lambda_{i+1}\left(\boldsymbol{x}_{0}^{*}\right)\right)=0 .
$$

Furthermore, according to the condition that $\boldsymbol{x}_{0}^{*}$ is a local minimizer of $\widehat{\Gamma}\left(\boldsymbol{x}_{0}\right)$, it follows that the derivative of $\widehat{\Gamma}$ with respect to $\boldsymbol{x}_{0}^{*}$ is zero. That is, according to (2.13),

$$
\left(\nabla_{\boldsymbol{x}_{0}} \lambda_{N}\right)^{T} \theta_{N}\left(\lambda_{N-1}, \lambda_{N}\right)=0 .
$$

Because $\nabla_{\boldsymbol{x}_{0}} \lambda_{N}$ is invertible, it follows that $\theta_{N}\left(\lambda_{N-1}, \lambda_{N}\right)=0$. Let $\boldsymbol{x}_{i}^{*}=\lambda_{i}\left(\boldsymbol{x}_{0}^{*}\right)$. It is then immediate that $\boldsymbol{x}_{0: N}^{*}$ satisfies (2.6) and is thus a stationary point of $\Gamma\left(\boldsymbol{x}_{0: N}\right)$. The proof is complete.

According to (2.15), the Hessian of $\widehat{\Gamma}$ at its local minimizer is only a lowerdimensional projection of the Hessian of $\Gamma$ at a corresponding point. Hence it is not necessary for the local minimum of $\widehat{\Gamma}$ to be the local minimum of $\Gamma$. Let us take a 
simple one-dimensional problem for a counterexample. Let $\Gamma\left(x_{0}, x_{1}\right)=x_{0} x_{1}-\frac{1}{2} x_{0}^{3}+$ $\frac{7}{2} x_{0}^{2}-6 x_{0}-3 x_{1}$. Here $N=1, \phi_{0}\left(x_{0}, x_{1}\right)=x_{0} x_{1}, \gamma_{0}\left(x_{0}\right)=-\frac{1}{2} x_{0}^{3}+\frac{7}{2} x_{0}^{2}-6 x_{0}$, and $\gamma_{1}\left(x_{1}\right)=-3 x_{1}$. It easy to show that $x_{1}=\frac{3}{2} x_{0}^{2}-7 x_{0}+6$ solves $\frac{\partial \Gamma\left(x_{0}, x_{1}\right)}{\partial x_{0}}=0$. By replacing $x_{1}$ in $\Gamma$ by $\frac{3}{2} x_{0}^{2}-7 x_{0}+6$, we can get $\widehat{\Gamma}\left(x_{0}\right)=x_{0}^{3}-8 x_{0}^{2}+21 x_{0}-18$. Obviously, $\left.\frac{\partial \widehat{\Gamma}}{\partial x_{0}}\right|_{x_{0}=3}=0$ and $\left.\frac{\partial^{2} \widehat{\Gamma}}{\partial x_{0}^{2}}\right|_{x_{0}=3}=2>0$; therefore, $x_{0}=3$ is the local minimizer of $\widehat{\Gamma}$. However, when $x_{0}=3$, the Hessian of $\Gamma$ satisfies

$$
\nabla_{x_{0}, x_{1}}^{2} \Gamma\left(x_{0}, x_{1}\right)=\left[\begin{array}{cc}
-3 x_{0}+7 & 1 \\
1 & 0
\end{array}\right]
$$

and is indefinite with eigenvalues -2.4142 and 0.4142 .

We can prove that the initial state of the local minimizer of (2.1) is also the local minimizer of (2.12). Moreover, and perhaps more important, we can now prove that the minimization of (2.12) is equivalent to a nonlinear equation with nonsingular Jacobian, whose residual can be computed by doing forward sweeps only.

THEOREM 3. Let $\boldsymbol{x}_{0}^{*}$ be the first component of a local minimizer of $\Gamma\left(\boldsymbol{x}_{0: N}\right)$ that satisfies the second-order sufficient condition. Then the following hold:

[i] $\boldsymbol{x}_{0}^{*}$ is a local minimizer of $\widehat{\Gamma}\left(\boldsymbol{x}_{0}\right)$ that satisfies the second-order sufficient conditions in $x_{0}$.

[ii] The matrix $\nabla_{\boldsymbol{x}_{0}} \lambda_{N}\left(x_{0}\right)$ is invertible at $\boldsymbol{x}_{0}^{*}$, where $\lambda_{N}\left(\boldsymbol{x}_{0}\right)$ is one of the mappings from Theorem 1.

[iii] In a neighborhood of $\boldsymbol{x}_{0}^{*}$, we have that

[iii-a] $\nabla_{\boldsymbol{x}_{0}} \theta_{N}\left(\lambda_{N-1}\left(\boldsymbol{x}_{0}\right), \lambda_{N}\left(\boldsymbol{x}_{0}\right)\right)$ is invertible, $\boldsymbol{x}_{0}^{*}$,

[iii-b] $\theta_{N}\left(\lambda_{N-1}\left(\boldsymbol{x}_{0}\right), \lambda_{N}\left(\boldsymbol{x}_{0}\right)\right)=0 \Rightarrow \boldsymbol{x}_{0}=\boldsymbol{x}_{0}^{*}$, and

[iii-c] there exists $C_{\theta}$ such that

$$
\left\|\theta_{N}\left(\lambda_{N-1}\left(\boldsymbol{x}_{0}\right) \lambda_{N}\left(\boldsymbol{x}_{0}\right)\right)\right\| \geq C_{\theta}\left\|\nabla_{x_{0}} \widehat{\Gamma}\left(x_{0}\right)\right\| .
$$

Proof. If $\boldsymbol{x}_{0: N}^{*}$ is a local minimizer of $\Gamma\left(\boldsymbol{x}_{0: N}\right)$, then $\boldsymbol{x}_{0: N}^{*}$ satisfies (2.9), and $\theta_{N}\left(\boldsymbol{x}_{N-1}, \boldsymbol{x}_{N}\right)=0$. Then, $\lambda_{i}\left(\boldsymbol{x}_{0}^{*}\right)=\boldsymbol{x}_{i}^{*}$ and $\nabla_{\boldsymbol{x}_{0}} \widehat{\Gamma}\left(\boldsymbol{x}_{0}^{*}\right)=0$.

Furthermore the second-order sufficient condition is satisfied by $x_{0: N}^{*}$ for $\Gamma$; in other words, $\nabla_{\boldsymbol{x}_{0: N} \boldsymbol{x}_{0: N}}^{2} \Gamma\left(\boldsymbol{x}_{0: N}^{*}\right)$ is positive definite. Then, $\nabla_{\boldsymbol{x}_{0} \boldsymbol{x}_{0}}^{2} \widehat{\Gamma}\left(\boldsymbol{x}_{0}^{*}\right)$ is also positive definite from (2.15) and the fact that the matrix $\Lambda$ in that equation is full rank because of the inclusion of an identity block.

To sum up, $\boldsymbol{x}_{0}^{*}$ is a stationary point of $\widehat{\Gamma}\left(\boldsymbol{x}_{0}^{*}\right)$ that satisfies the second-order sufficient condition. Then, it is also a local minimizer of $\widehat{\Gamma}$, and part [i] of the Theorem is proved.

For part [ii], we use (2.13) to obtain that (where we drop the dependence of $\lambda_{i}$ on $x_{0}$ to simplify notation)

$$
\begin{aligned}
\nabla_{\boldsymbol{x}_{0} \boldsymbol{x}_{0}}^{2} \widehat{\Gamma}\left(\boldsymbol{x}_{0}^{*}\right)=( & \left(\nabla_{\boldsymbol{x}_{0}} \lambda_{N}\right)^{T} \nabla_{\lambda_{N-1}} \theta_{N}\left(\lambda_{N-1}, \lambda_{N}\right) \nabla_{\boldsymbol{x}_{0}} \lambda_{N-1} \\
& +\left(\nabla_{\boldsymbol{x}_{0}} \lambda_{N}\right)^{T} \nabla_{\lambda_{N}} \theta_{N}\left(\lambda_{N-1}, \lambda_{N}\right) \nabla_{\boldsymbol{x}_{0}} \lambda_{N} .
\end{aligned}
$$

Note that the component of the Hessian involving the second-derivative $\lambda_{N}$ disappears since $\theta_{N}\left(\lambda_{N-1}, \lambda_{N}\right)=0$ at $\boldsymbol{x}_{0}^{*}$. Also note that the above formula for $\nabla_{\boldsymbol{x}_{0} \boldsymbol{x}_{0}}^{2} \widehat{\Gamma}\left(\boldsymbol{x}_{0}^{*}\right)$ does not imply that it is nonsymmetric (which would be a contradiction). The symmetry of the matrix would eventually unfold after using the recursion for $\lambda_{i}$ and, implicitly, their Jacobians. Nevertheless, the form presented is sufficient for us to reach our conclusions. 
Assume now that $\nabla_{\boldsymbol{x}_{0}} \lambda_{N}$ are not invertible. Then, there must be a vector $u \neq 0$ such that $\nabla_{\boldsymbol{x}_{0}} \lambda_{N} u=0$. Using (2.18), we obtain that $u^{T} \nabla_{\boldsymbol{x}_{0} \boldsymbol{x}_{0}}^{2} \widehat{\Gamma}\left(\boldsymbol{x}_{0}^{*}\right) u=0$, which contradicts the conclusion reached in part [i]. This proves part [ii] of the theorem.

For part [iii], we use (2.13) to obtain

$$
\left(\nabla_{\boldsymbol{x}_{0}} \lambda_{N}\right)^{-T} \nabla_{\boldsymbol{x}_{0}} \widehat{\Gamma}\left(\boldsymbol{x}_{0}\right)=\theta_{N}\left(\lambda_{N-1}, \lambda_{N}\right),
$$

which in turn, with $\nabla_{\boldsymbol{x}_{0}} \widehat{\Gamma}\left(\boldsymbol{x}_{0}^{*}\right)=0$, results in

$$
\nabla_{\boldsymbol{x}_{0}} \theta_{N}\left(\lambda_{N-1}\left(\boldsymbol{x}_{0}^{*}\right), \lambda_{N}\left(\boldsymbol{x}_{0}^{*}\right)\right)=\left(\nabla_{\boldsymbol{x}_{0}} \lambda_{N}\right)^{-T}\left(\boldsymbol{x}_{0}^{*}\right) \nabla_{\boldsymbol{x}_{0} \boldsymbol{x}_{0}}^{2} \widehat{\Gamma}\left(\boldsymbol{x}_{0}^{*}\right)
$$

Since the latter relationship - following parts [i] and [ii] — is a multiplication between two nonsingular matrices, it follows that $\nabla_{\boldsymbol{x}_{0}} \theta_{N}\left(\lambda_{N-1}\left(\boldsymbol{x}_{0}^{*}\right), \lambda_{N}\left(\boldsymbol{x}_{0}^{*}\right)\right)$ is nonsingular, which proves [iii-a]. From Assumption $1, \nabla_{\boldsymbol{x}_{0}} \theta_{N}(\cdot, \cdot)$ is a continuous function, whereas from Theorem 1 we have that $\lambda_{N}(\cdot)$ and $\lambda_{N-1}(\cdot)$ are continuous functions, which implies that the mapping $\nabla_{\boldsymbol{x}_{0}} \theta_{N}\left(\lambda_{N-1}\left(\boldsymbol{x}_{0}\right), \lambda_{N}\left(\boldsymbol{x}_{0}\right)\right)$ is continuous in a neighborhood of $\boldsymbol{x}_{0}^{*}$. From [iii-a] the mapping is nonsingular at $\boldsymbol{x}_{0}^{*}$ and, since it is continuous, it is nonsingular in a neighborhood of $\boldsymbol{x}_{0}^{*}$, which implies local uniqueness and thus [iii-b]. From (2.19) and part [ii], the conclusion of [iii-c] follows as well, as $\nabla_{\boldsymbol{x}_{0}} \lambda_{N}$ is continuous and thus invertible in a neighborhood of $\boldsymbol{x}_{0}^{*}$.

This completes the proof of part [iii] and of the theorem.

2.4. Second-order sufficient conditions for the weakly constrained 4DVar problem. We now investigate under what circumstances the $4 \mathrm{D}$-Var problem satisfies the second-order sufficient conditions that are the main requirement of the key result, Theorem 3. We first find the Hessian matrix of $\Gamma$. Define

$$
\begin{aligned}
W_{i}:= & \left(\nabla_{\boldsymbol{x}_{i}} \mathcal{H}_{i}\left(\boldsymbol{x}_{i}\right)\right)^{T} R_{i}^{-1} \nabla_{\boldsymbol{x}_{i}} \mathcal{H}_{i}\left(\boldsymbol{x}_{i}\right) \\
& +\left(\left(\left(\boldsymbol{x}_{i+1}-\mathcal{M}_{i}\left(\boldsymbol{x}_{i}\right)\right)^{T} Q_{i}^{-T}\right) \bigotimes I_{s}\right) \nabla_{\boldsymbol{x}_{i}} \operatorname{vec}\left(\left(\nabla_{\boldsymbol{x}_{i}} \mathcal{M}_{i}\left(\boldsymbol{x}_{i}\right)\right)^{T}\right) \\
& +\left(\left(\left(\boldsymbol{y}_{i}-\mathcal{H}_{i}\left(\boldsymbol{x}_{i}\right)\right)^{T} R_{i}^{-T}\right) \bigotimes I_{m}\right) \nabla_{\boldsymbol{x}_{i}} \operatorname{vec}\left(\left(\nabla_{\boldsymbol{x}_{i}} \mathcal{H}_{i}\left(\boldsymbol{x}_{i}\right)\right)^{T}\right)
\end{aligned}
$$

for $i=0, \ldots, N$. Define $S$ to be a symmetric block tridiagonal matrix with $V_{i}$, $i=0, \ldots, N$, as diagonal and $-U_{i}, i=0, \ldots, N-1$, as subdiagonal, and

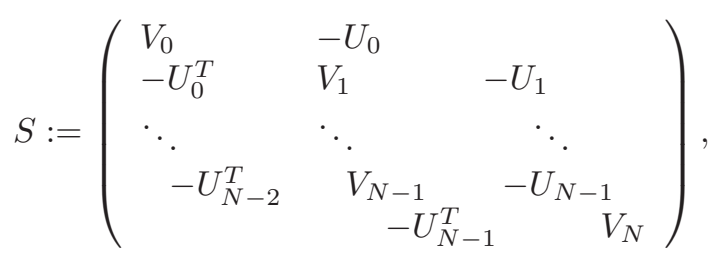

where

$$
\begin{aligned}
& U_{i}:=\left(\nabla_{\boldsymbol{x}_{i}} \mathcal{M}_{i}\left(\boldsymbol{x}_{i}\right)\right)^{T} Q_{i}^{-1}, \quad i=0, \ldots, N-1, \\
& V_{i}:=Q_{i-1}^{-1}+\left(\nabla_{\boldsymbol{x}_{i}} \mathcal{M}_{i}\left(\boldsymbol{x}_{i}\right)\right)^{T} Q_{i}^{-1} \nabla_{\boldsymbol{x}_{i}} \mathcal{M}_{i}\left(\boldsymbol{x}_{i}\right), \quad i=0, \ldots, N-1, \\
& V_{0}:=Q_{B}^{-1}+\left(\nabla_{\boldsymbol{x}_{0}} \mathcal{M}_{0}\left(\boldsymbol{x}_{0}\right)\right)^{T} Q_{0}^{-1} \nabla_{\boldsymbol{x}_{0}} \mathcal{M}_{0}\left(\boldsymbol{x}_{0}\right), \quad V_{N}:=Q_{N-1}^{-1} .
\end{aligned}
$$

Because $\Gamma$ takes the special form as in (2.1)-(2.4), its Hessian matrix is a block tridiagonal matrix. We can verify that $U_{i}=-N \nabla_{\boldsymbol{x}_{i}} \nabla_{\boldsymbol{x}_{i+1}} \Gamma\left(\boldsymbol{x}_{0: N}\right), V_{i}+W_{i}=N \nabla_{\boldsymbol{x}_{i}}^{2} \Gamma\left(\boldsymbol{x}_{0: N}\right)$ 
as follows:

$$
\nabla_{\boldsymbol{x}_{0: N}}^{2} \Gamma\left(\boldsymbol{x}_{0: N}\right)=\frac{1}{N}\left(S+\operatorname{diag}\left(W_{0}, \ldots, W_{N}\right)\right)
$$

where $S$ is defined by $(2.20)$ and (2.23).

Lemma 1. Suppose that the first- and second-order derivatives of $\mathcal{M}_{i}$ and $\mathcal{H}_{i}$ are bounded; $\nabla_{x} \mathcal{M}_{i}$ is nonsingular; $Q_{i}, R_{i}, Q_{B}$ are positive definite (all of which are standard $4 D$-Var conditions); and $W_{i}$ are positive semidefinite at the solution $\boldsymbol{x}_{0: N}^{*}$, $i=0,1, \ldots, N$. Then $\nabla_{\boldsymbol{x}_{0: N}}^{2} \Gamma\left(\boldsymbol{x}_{0: N}\right)$ is positive definite at that solution.

The significance of this result is that the optimization problem of the weakly constrained 4D-Var satisfies the second-order sufficient condition. Therefore, Theorem 3 [iii] applies to ensure that the solution of the 4D-Var problem satisfies a nonlinear equation defined by $\theta_{N}$ and the mappings $\lambda$, a fact which we will exploit in section 2.5 to create a low-memory method to find a local minimum of (1.1).

Of all the conditions invoked, only the one concerning the positive definiteness of $W_{i}$ is nonstandard. They hold, for example, for linear systems or for the case where the model and observation error is 0 at the solution. Note, however, that these conditions are sufficient but not necessary for well-posedness of the nonlinear equation (2.25). The only necessary condition is the second-order condition, though it is of course difficult to ensure a priori in all nonlinear problems for any variational approach, including ours.

Proof. If $Z \neq 0$, then

$$
Z^{T} S Z=\boldsymbol{z}_{0}^{T} Q_{B}^{-1} \boldsymbol{z}_{0}+\sum_{i=0}^{N-1}\left(\nabla_{\boldsymbol{x}_{i}} \mathcal{M}_{i}\left(\boldsymbol{x}_{i}\right) \boldsymbol{z}_{i}-\boldsymbol{z}_{i+1}\right)^{T} Q_{i}^{-1}\left(\nabla_{\boldsymbol{x}_{i}} \mathcal{M}_{i}\left(\boldsymbol{x}_{i}\right) \boldsymbol{z}_{i}-\boldsymbol{z}_{i+1}\right)>0 .
$$

The inequality holds because if the right-hand side is 0 , then $\boldsymbol{z}_{i+1}=\left(\nabla_{\boldsymbol{x}_{i}} \mathcal{M}_{i}\left(\boldsymbol{x}_{i}\right)\right) \boldsymbol{z}_{i}$ and $\boldsymbol{z}_{0}=0$, which in turn implies $Z=0$, a contradiction. If $W_{i}$ is positive semidefinite, then the Hessian matrix of $\Gamma,(2.24)$, is positive definite, and the proof is complete.

2.5. Our low-memory approach. The essence of our approach follows from Theorems 1 and 3. From these theorems, the minimizer $x_{0}^{*}$ of (2.12) and, implicitly, the first component of the minimizer of the target function (2.1), can be obtained by solving the nonlinear systems of equations in $\boldsymbol{x}_{0}$ :

$$
\theta_{N}\left(\lambda_{N-1}\left(\boldsymbol{x}_{0}\right), \lambda_{N}\left(\boldsymbol{x}_{0}\right)\right)=0 .
$$

In the case of the 4D-Var functional (1.1), using (2.5c) with the definition (2.2), with $\boldsymbol{x}_{N-1}, \boldsymbol{x}_{N}$ computed by recurrence (2.11), we have that

$$
\begin{aligned}
\theta_{N}\left(\boldsymbol{x}_{N-1}, \boldsymbol{x}_{N}\right)= & 2 Q_{N-1}^{-1}\left(\boldsymbol{x}_{N}-\mathcal{M}_{N-1}\left(\boldsymbol{x}_{N-1}\right)\right) \\
& -2\left(\nabla_{\boldsymbol{x}_{N}} \mathcal{H}_{N}\left(\boldsymbol{x}_{N}\right)\right)^{T} R_{N}^{-1}\left(\boldsymbol{y}_{N}-\mathcal{H}_{N}\left(\boldsymbol{x}_{N}\right)\right) .
\end{aligned}
$$

For given $\boldsymbol{x}_{0}$, the function on the left of (2.25) is evaluated by computing $\lambda_{i}\left(\boldsymbol{x}_{0}\right)$ recursively using Theorem 1. In turn, the nonlinear equation (2.25) is well-posed from Theorem 3[iii]. The resulting nonlinear equation can now be solved by limitedmemory quasi-Newton nonlinear equation methods such as limited-memory Broyden methods $[27,4]$. Alternatively, under some conditions, the same recursion can be used to compute the objective function (2.12) and a descent direction for it, as we 
will illustrate in section 2.7. In turn, this can be used in a limited-memory quasiNewton optimization approach such as limited-memory BFGS (L-BFGS) [4, 15].

Therefore, in principle, $(2.25)$ can be solved by using only $\mathcal{O}(1)$ stored vectors. The only vectors that need to be stored are the current $\boldsymbol{x}_{0}$, the vectors at the current recursion step $\left(\boldsymbol{x}_{i}\right.$ and $\boldsymbol{x}_{i+1}$ at the $i$ th step of the recursion in Theorem 1 ), and the vectors needed by the limited-memory Broyden (L-Broyden) method. Once the convergence criterion is satisfied, the sought-after quantity (typically, the best estimate of the last state $x_{N}^{*}$ ) can be output after one more recursion.

In any case, our approach compares favorably with a brute-force minimization of (2.1) where, in principle, all vectors $\boldsymbol{x}_{i}$ need to be stored, $i=0,1,2, \ldots, N$. For high-fidelity simulations in memory-starved environments, as the emerging high-end computing facilities appear to be, this can be a major handicap.

2.6. Comparison with the strong constraint case. Some of the difficulties in the direct approach to (2.1) appear in the case with strong constraints:

$$
\begin{aligned}
& \min \Gamma\left(\boldsymbol{x}_{0: N}\right):=\frac{1}{N}\left(\sum_{i=0}^{N-1}\left[\gamma_{i}\left(\boldsymbol{x}_{i}\right)+\phi_{i}\left(\boldsymbol{x}_{i}, \boldsymbol{x}_{i+1}\right)\right]+\gamma_{N}\left(\boldsymbol{x}_{N}\right)\right), \\
& \boldsymbol{x}_{i+1}=M_{i}\left(\boldsymbol{x}_{i}\right), \quad i=0,1,2, \ldots, N-1 .
\end{aligned}
$$

Note that, because of the constraints, this new problem has only 1 vector degree of freedom, whereas the problem of minimizing (2.1) had $N+1$ degrees of freedom. In the $4 \mathrm{D}$-Var case with strong constraints, as applied operationally, the terms $\phi_{i}$ do not appear, but we preserve them for generality; they will not change our approach.

The optimality conditions for (2.27) can be obtained by introducing Lagrange multipliers $\boldsymbol{m}_{i}, i=0,1, \ldots, N-1$, and the Lagrangian function

$$
\mathcal{L}\left(\boldsymbol{x}_{0: N}, \boldsymbol{m}_{0: N-1}\right)=\Gamma\left(\boldsymbol{x}_{0: N}\right)+\sum_{i=0}^{N-1}\left(\boldsymbol{x}_{i+1}-\mathcal{M}_{i}\left(x_{i}\right)\right)^{T} \boldsymbol{m}_{i} .
$$

The optimality-feasibility conditions become $\nabla_{\boldsymbol{x}_{i}} \mathcal{L}=0, i=0,1, \ldots, N, \nabla_{\boldsymbol{m}_{i}} \mathcal{L}=0$, $i=0,1, \ldots, N-1$. That is,

$$
\begin{aligned}
0= & \nabla_{\boldsymbol{x}_{0}} \gamma\left(\boldsymbol{x}_{0}\right)+\nabla_{\boldsymbol{x}_{0}} \phi_{0}\left(\boldsymbol{x}_{0}, \boldsymbol{x}_{1}\right)-\nabla_{\boldsymbol{x}_{0}} \mathcal{M}_{0}^{T}\left(\boldsymbol{x}_{0}\right) \boldsymbol{m}_{0}, \\
0= & \nabla_{\boldsymbol{x}_{i}} \gamma\left(\boldsymbol{x}_{i}\right)+\nabla_{\boldsymbol{x}_{i}} \phi_{i-1}\left(\boldsymbol{x}_{i-1}, \boldsymbol{x}_{i}\right)+\nabla_{\boldsymbol{x}_{i}} \phi_{i}\left(\boldsymbol{x}_{i}, \boldsymbol{x}_{i+1}\right) \\
& \quad+\boldsymbol{m}_{i-1}-\nabla_{\boldsymbol{x}_{i}} \mathcal{M}_{i}^{T} \boldsymbol{m}_{i}, \quad i=1,2, \ldots, N-1, \\
0= & \nabla_{\boldsymbol{x}_{N}} \gamma\left(\boldsymbol{x}_{N}\right)+\nabla_{\boldsymbol{x}_{N}} \phi_{N-1}\left(\boldsymbol{x}_{N-1}, \boldsymbol{x}_{N}\right)+\boldsymbol{m}_{N-1}, \\
0= & \boldsymbol{x}_{i+1}-\mathcal{M}_{i}\left(\boldsymbol{x}_{i}\right), \quad i=0,1, \ldots, N-1 .
\end{aligned}
$$

We are now faced with two options. The first is the classical adjoint approach, which can be thought to follow from Pontryagin's principle of optimal control. That is, one can think of $\boldsymbol{x}_{0}$ as the only (vector) degree of freedom.

Indeed, this setup is identical to the optimal discrete nonlinear control setup [3, Proposition 3.2]. It can be seen from that reference that the situation described here corresponds to the case in which the control over the first time stage is the initial state variable, $\boldsymbol{x}_{0}$, and the dynamics and the objective function for the other variables do not depend on the control. Following the maximum principle in this setup, at a given $\boldsymbol{x}_{0}$, one computes the states by carrying out the forward recursion $(2.29 \mathrm{~d})$ and stores them. Subsequently, the Lagrange multiplier $\boldsymbol{m}_{N-1}$ is computed from (2.29c). Then, 
all other Lagrange multipliers (the "adjoint variables") are computed recursively from (2.29b) backward all the way to $\boldsymbol{m}_{0}$. Next, the quantity

$$
\nabla_{\boldsymbol{x}_{0}} \mathcal{L}=\nabla_{\boldsymbol{x}_{0}} \gamma\left(\boldsymbol{x}_{0}\right)+\nabla_{\boldsymbol{x}_{0}} \phi_{0}\left(\boldsymbol{x}_{0}, \boldsymbol{x}_{1}\right)-\nabla_{\boldsymbol{x}_{0}} \mathcal{M}_{0}^{T}\left(\boldsymbol{x}_{0}\right) \boldsymbol{m}_{0}
$$

is evaluated. This is simply the derivative of the objective function restricted on the feasible manifold defined by $(2.29 \mathrm{~d})$ but unrestricted in $\boldsymbol{x}_{0}$.

Subsequently, since the gradient is available, one has the option of carrying out a quasi-Newton optimization approach or, similar to the weakly constrained case described before, of solving the nonlinear equation resulting from setting the gradient to zero, that is, (2.29a). Nevertheless, note that to carry out the backward recursion, as is the case with all adjoint approaches, one needs to store at some point all vectors $x_{0: N}$, which may be a significant cost.

Alternatively, and related to the approach in this work, one can look at the optimality-feasibility conditions (2.29) as the nonlinear equation

$$
\nabla_{\boldsymbol{x}_{N}} \gamma\left(\boldsymbol{x}_{N}\left(\boldsymbol{x}_{0}\right)\right)+\nabla_{\boldsymbol{x}_{N}} \phi_{N-1}\left(\boldsymbol{x}_{N-1}\left(\boldsymbol{x}_{0}\right), \boldsymbol{x}_{N}\left(\boldsymbol{x}_{0}\right)\right)+\boldsymbol{m}_{N-1}\left(\boldsymbol{x}_{0}\right)=0 .
$$

Here, the component functions of $\boldsymbol{x}_{0}$ are defined recursively as follows. From a prescribed $\boldsymbol{x}_{0},(2.29 \mathrm{~d})$ is solved for $i=0$, and $\boldsymbol{x}_{1}\left(\boldsymbol{x}_{0}\right)$ is obtained. Subsequently, (2.29a) is solved for $\boldsymbol{m}_{0}\left(\boldsymbol{x}_{0}\right)$, which exists uniquely if $\nabla_{x} \mathcal{M}_{0}\left(\boldsymbol{x}_{0}\right)$ is invertible (which is the case for all time resolvents). Then a recursion is carried out through (2.29b) and (2.29d), obtaining at each step $\boldsymbol{x}_{i+1}\left(\boldsymbol{x}_{0}\right)$ and $\boldsymbol{m}_{i}\left(\boldsymbol{x}_{0}\right)$ up to $i=N-1$. At that point, all the elements needed to evaluate the left-hand side of (2.30) are computed, and that quantity can be evaluated. At this point, one can apply the L-Broyden method and carry out the solution of the optimality system with $\mathcal{O}(1)$ vector storage as in the weakly constrained session.

On the other hand, the case for using the nonlinear equation-limited-memory method for the strongly constrained case -is less compelling, since for the adjoint case, $\mathcal{O}(\log N)$ vector storage schemes exist by using checkpointing on the adjoint calculation while regenerating the $\boldsymbol{x}$ vectors as needed from $(2.29 \mathrm{~d})$. While this results in substantial additional computational expense, the approach is well understood and has the advantage of leading to an optimization problem and guarantees of global convergence to stationary points. Moreover, one does not need an extra solve with $\nabla_{\boldsymbol{x}} \mathcal{M}(\boldsymbol{x})$ at every step. Otherwise, in terms of conceptual complexity, the limitedmemory quasi-Newton approach for the adjoint-optimization approach seems to be comparable to the limited-memory quasi-Newton approaches proposed in this work.

In the weakly constrained case considered here (2.1), however, the backward recursion option does not seem to exist. The reason is that the problem is now truly a problem over an $(N+1) d$-dimensional space defined by $\boldsymbol{x}_{0: N}$, as opposed to over a $d$-dimensional case defined by $\boldsymbol{x}_{0}$ in the strongly constrained case. Therefore there is no projected gradient to speak of, which is an important concept in adjoint calculations. One could consider the optimality conditions of Theorem 1 as constraints on (2.1) and then apply the approach described through (2.29). Doing so, however, would require second derivatives of $M_{i}(x)$, which seems a steep price to pay for a first-order algorithm insofar as optimization properties are concerned. Therefore our approach in section 2.3, while related to Pontryagin's maximum principle, cannot be really inferred from it. We will thus concentrate on the algorithm described in section 2.3 .

The comparison with the strongly constrained case reveals another interesting insight. In the control literature, the forward-nonlinear equation approach is thought 
of as a shooting approach for a boundary value problem. We can thus think of the approach from this work as a shooting approach for the nonlinear equation of the optimality conditions of (2.1) combined with a quasi-Newton method.

2.7. Optimization-based low-memory approach. Here we investigate the possibility of obtaining a descent direction for $\widehat{\Gamma}$ by doing forward sweeps only. The advantage of such an approach compared to an adjoint one is that no information needs to be stored for a reverse sweep, which ensures a low-memory behavior. The aim is to find a vector which is guaranteed to have a positive inner product with $\nabla_{\boldsymbol{x}_{0}} \widehat{\Gamma}$. In turn, this would provide a theoretical basis for using optimization algorithms using line search and positive definite approximations of the Hessian matrix, as is the case for limited-memory, optimization-based quasi-Newton methods such as L-BFGS methods [15].

We prove the main results for optimization-based approaches below.

Lemma 2. Suppose that Assumption [A] in section 3 holds and that the sequence $\boldsymbol{x}_{i}, i=1, \ldots, N$, is derived by the recurrence formula (2.11), and $\theta_{N}$ is computed by (2.26). Then there exist $a T_{\delta}$ and an $N_{0}$ such that $\nabla_{\boldsymbol{x}_{0}} \boldsymbol{x}_{N}$ is positive definite for $T<T_{\delta}$ and $N \geq N_{0}$.

Proof. We use the same notations as in the proof of Theorem 5. Following (3.12) and invoking Lemma 7 we obtain that $G_{N}=\nabla_{\boldsymbol{x}_{0}} \boldsymbol{x}_{N}$ satisfies $\left\|G_{N}-I_{s}\right\| \rightarrow$ $\left\|\exp (P T)-I_{s}\right\|$. Choose now $T_{\delta}$ such that $\left\|\exp (P T)-I_{s}\right\| \leq \frac{1}{4}$ for all $T \leq T_{\delta}$. Then, from the preceding limit, there exists $N_{0}$ such that $\left\|G_{N}-I_{s}\right\| \leq \frac{1}{3}$ for all $N \geq N_{0}$. Since this implies that $\left\|G_{N}^{T}-I_{s}\right\| \leq \frac{1}{3}$, it follows that $\left\|\frac{G_{N}^{T}+G_{N}}{2}-I_{s}\right\| \leq \frac{1}{3}$, and thus $G_{N}^{T}+G_{N}$ is symmetric and positive definite, and so is $G_{N}$. This proves the claim.

Theorem 4. Suppose Assumption [A] holds and the sequence $\boldsymbol{x}_{i}, i=1,2, \ldots, N$, is derived by the recurrence formula (2.11), and $\theta_{N}$ is computed by (2.26). Then there exists a $T_{\delta}$ such that $\left(\nabla_{\boldsymbol{x}_{0}} \widehat{\Gamma}\right)^{T} \theta_{N}$ is positive for $T<T_{\delta}$.

Proof. From (2.13), we have

$$
\left(\nabla_{\boldsymbol{x}_{0}} \widehat{\Gamma}\right)^{T} \theta_{N}=\left(\theta_{N}\right)^{T}\left(\nabla_{\boldsymbol{x}_{0}} \boldsymbol{x}_{N}\right) \theta_{N} .
$$

According to Lemma 2, there exists a $T_{\delta}$ such that $\nabla_{\boldsymbol{x}_{0}} \boldsymbol{x}_{N}$ is positive definite for $T<T_{\delta}$. Hence the proof is complete.

The significance of the result of Theorem 4 is that scaling the vector $\theta_{N}$ obtained from the forward recursion (2.9) will now provide a descent direction for $\widehat{\Gamma}\left(\boldsymbol{x}_{0}\right)$ when the time interval is small enough under the conditions described in the theorem.

Of course, the condition $T \leq T_{\delta}$ may be quite limiting. On the other hand, as proved in Theorem $3\left[\right.$ iii-c] we have that $\theta_{N}$ will be proportional with the distance from the current point to the solution. Therefore its size is proportional to that of the gradient, and while we cannot ensure it will provide a descent direction, it is quite likely that either it or its reciprocal will provide a substantial descent. So we will use it even for $T$ larger than Theorem 4, with the expectation that it could work well, even though this cannot be generally proved.

3. Stability issues. The advantage of our method is most evident at large $N$ values. On the other hand, in that regime the recursive nature of the solution opens the door to having an unstable scheme that, even if formally well defined, results in quantities too large to be practical. These difficulties are not by themselves unique to our method; the recurrence in the maximum principle approach (adjoint approach) in the case of strong constraints is also susceptible to instability if the number of steps 
considered is too large in relationship to the size of the eigenvalues of $\nabla_{x} \mathcal{M}(\boldsymbol{x})[3$, equation (3.38)].

Therefore, the stability of the recurrence (2.11) needs to be studied. We are particularly interested in the limit case $N \rightarrow \infty$. For a dynamical system such as Burgers' equation, given a fixed time interval, it is desirable that when the time step goes to zero (i.e., the iteration number $N$ increases to infinite) and the time interval $T$ is fixed, the solution of (2.11) remains bounded.

Since a complete analysis is difficult for nonlinear systems, we will carry out this analysis for linear time-invariant systems. That is, we will investigate only the case of linear $\mathcal{M}_{i}$ in $(2.2)$ and $\mathcal{H}_{i}$ from (2.3):

$$
\begin{aligned}
& \mathcal{M}_{i}\left(\boldsymbol{x}_{i}\right)=A \boldsymbol{x}_{i}, \\
& \mathcal{H}_{i}\left(\boldsymbol{x}_{i}\right)=B \boldsymbol{x}_{i} .
\end{aligned}
$$

By replacing (3.1) and (3.2) in (2.11), the recurrence formula for computing $\boldsymbol{x}_{i+1}$, $i=1, \ldots, N-1$, becomes

$$
\begin{aligned}
\boldsymbol{x}_{i+1}= & -Q A^{-T} B^{T} R^{-1} \boldsymbol{y}_{i}-Q A^{-T} Q^{-1} A \boldsymbol{x}_{i-1} \\
& +\left(Q A^{-T} Q^{-1}+A+Q A^{-T} B^{T} R^{-1} B\right) \boldsymbol{x}_{i},
\end{aligned}
$$

and

We want to allow for an asymptotic analysis with $h=\frac{T}{N} \rightarrow 0$, and with $T$ fixed. We thus discuss how the various quantities of interest should behave with $h$. In the following we use the Landau notation: $a=\mathcal{O}(h)$ and, respectively, $a=o(h)$ indicate that $\|a / h\|$ is bounded and, respectively, converges to 0 as $h \rightarrow 0$.

To mimic the discretization of a continuous dynamical system, the propagator of the dynamical system should satisfy $A=I+\mathcal{O}(h)=I+\mathcal{O}\left(\frac{T}{N}\right)$. Since the covariance matrix $R$ models instrument error, it is reasonable to assume that it is independent of the time step, and we will thus take it to be constant. About the numerical error model, consistency requires that the error be no larger than $\mathcal{O}(h)=\mathcal{O}\left(\frac{T}{N}\right)$, the size of the time step, and thus its variance to be no larger than the square of it. We make a marginally stronger assumption below.

Assumption [A]. We assume that $A=A(h)=I+h P+\mathcal{O}\left(h^{2}\right), Q=Q(h)=$ $\psi(h)\left(Q_{0}+\mathcal{O}(h)\right), R=\mathcal{O}(1), Q_{B}=\mathcal{O}(1)$ for $h \rightarrow 0$. Here $Q_{0}$ is a constant invertible covariance matrix, and $\psi(h)=o\left(h^{2}\right)$. Here $h=T / N$, and $N$ is the number of time intervals considered in the system.

To carry out the stability analysis under these circumstances, we first prove the following lemma. Note that $A$ and $Q$ depend on $h$.

Lemma 3. Under Assumption $[\mathrm{A}],\left\|Q A^{-T} Q^{-1}\right\|^{N},\left\|Q A^{T} Q^{-1}\right\|^{N}$, and $\|A\|^{N}$ are bounded for all $h$ sufficiently small.

Proof. See section A.1.

Note that the second-order recurrence in (3.3) can be written in a matrix-vector form as

$$
\left(\begin{array}{c}
\boldsymbol{x}_{i+1} \\
\boldsymbol{x}_{i}
\end{array}\right)=L\left(\begin{array}{c}
\boldsymbol{x}_{i} \\
\boldsymbol{x}_{i-1}
\end{array}\right)+S\left(\begin{array}{c}
\boldsymbol{y}_{i} \\
0
\end{array}\right)
$$

where

$$
L:=\left(\begin{array}{cc}
D & -E \\
I_{s} & \mathbf{0}
\end{array}\right)
$$


$E:=Q A^{-T} Q^{-1} A, I_{s}$ is the $s \times s$ identity matrix, and $D:=Q A^{-T} Q^{-1}+A+$ $Q A^{-T} B^{T} R^{-1} B$. Clearly $L$ is a matrix with special form, and we can derive $L^{N}$ with some extra effort. Our first attempt is made in the following lemma.

Lemma 4. Let $U$ and $V$ be $s \times s$ square matrices, and

$$
L:=\left(\begin{array}{cc}
U+V & -U V \\
I_{s} & 0
\end{array}\right)
$$

Then

$$
L^{n}=\left(\begin{array}{cc}
g_{n} & -g_{n-1} U V \\
g_{n-1} & g_{n}-g_{n-1}(U+V)
\end{array}\right)
$$

where $g_{n}=\sum_{i=0}^{n} V^{i} U^{n-i}$.

Proof. See section A.2.

However, the matrix $L$ in Lemma 4 is still a bit different in our case, which we begin to investigate with the following lemma.

Lemma 5. Let $U, V$, and $C$ be $s \times s$ square matrices, and

$$
L:=\left(\begin{array}{cc}
U+V+C & -U V \\
I_{s} & 0
\end{array}\right) .
$$

Then

$$
L^{n}=\left(\begin{array}{cc}
f_{n} & -f_{n-1} U V \\
f_{n-1} & -f_{n-2} U V
\end{array}\right)
$$

where $f_{n}(U+V+C)-f_{n-1} U V=f_{n+1}$, with $f_{-1}=\mathbf{0}_{s}, f_{0}=I_{s}$.

Proof. See section A.3.

The difficulty with Lemma 5 is that the term $C$ makes a general solution for $f_{n}$ very complicated algebraically. To reduce the calculation of $f_{n}$ to the calculation of $g_{n}$, we prove the following.

LEMMA 6. Let $J_{1}(h), J_{2}(h) \in \mathbb{R}^{s \times s}$ be matrices satisfying $J_{1}(h)=J_{1}^{0}+\mathcal{O}(h)$, $J_{2}(h)=J_{2}^{0}+\mathcal{O}(h)$ such that $J_{1}^{0}$ and $-J_{2}^{0}$ have no common eigenvalues and $C(h) \in$ $\mathbb{R}^{s \times s}, C(h)=o\left(h^{2}\right)$. Define the matrices $U(h)=I_{s}+h J_{1}(h), V(h)=I_{s}-h J_{2}(h)$. There exists $h_{0}$ such that for all $0 \leq h \leq h_{0}$ there exist the matrices $\widehat{U}(h)$ and $\widehat{V}(h)$ satisfying

$$
\widehat{U}(h)+\widehat{V}(h)=C(h)+U(h)+V(h), \quad \widehat{U}(h) \widehat{V}(h)=U(h) V(h)
$$

and

$$
\|\widehat{U}(h)-U(h)\|=o(h), \quad\|\widehat{V}(h)-V(h)\|=o(h) .
$$

Proof. See section A.4.

The key bounding calculation is now provided by the following lemma.

LEMma 7. Let $f_{n}$ be the sequence from Lemma 5 as applied to (3.5)-(3.6). To this end, we use the identifications $U=Q A^{-T} Q^{-1}, V=A$, and $C=Q A^{-T} B^{T} R^{-1} B$. Assume that Assumption $[\mathrm{A}]$ holds and that $P^{T}$ and $-P$ have no common eigenvalues. Then the following hold:

[i] $\frac{1}{N}\left\|f_{n}\right\|$ is bounded for all $N$ and $1 \leq n \leq N$.

[ii] For any $\epsilon$ there exists $N_{0}$ such that for all $N \geq N_{0}$, we have that

$$
\left\|f_{N}-f_{N-1} Q A^{-T} Q^{-1}\right\|<\left\|e^{P T}-I_{s}\right\|+\epsilon .
$$

Note that $Q, A$ depend on $h=\frac{T}{N}$ as defined in Assumption [A]. 
Proof. See section A.5.

Remark. The only assumption we made beyond Assumption $[\mathrm{A}]$ is that $P$ and $-P^{T}$ have no common eigenvalues. This is the case, for example, if $A$ is the propagator of the dynamical system $\frac{d x}{d t}=P x$, where $P$ is a stable matrix. Therefore, the condition is satisfied if the target system is stable.

TheOREM 5. Suppose that the sequence $\boldsymbol{x}_{i}, i=1, \ldots, N$, is derived by recurrence formula (2.11), and $\theta_{N}$ is computed by (2.26). Then $\left\|\nabla_{\boldsymbol{x}_{0}} \boldsymbol{x}_{N}\right\|$ is bounded as $N \rightarrow \infty$, and thus the recurrence (3.3) is stable.

Proof. We first prove that

$$
\nabla_{\boldsymbol{x}_{0}} \boldsymbol{x}_{N}=f_{N}+f_{N-1}\left(-Q A^{-T} Q^{-1}+Q A^{-T} Q_{B}^{-1}\right),
$$

where $f_{n}$ is defined as in Lemma 5 .

The second-order recurrence (3.3) can be written as (3.5) with $L$ defined as in (3.6). Let $L_{1}^{N}, L_{2}^{N}, L_{3}^{N}$, and $L_{4}^{N}$ denote the upper left block, upper right block, bottom left block, and bottom right block of $L^{N}$, respectively. According to (3.5), we will have

$$
\nabla_{\boldsymbol{x}_{0}} \boldsymbol{x}_{N}=L_{3}^{N} \frac{\partial \boldsymbol{x}_{1}}{\boldsymbol{x}_{0}}+L_{4}^{N}=L_{3}^{N}\left(Q A^{-T} Q_{B}^{-1}+A+Q A^{-T} B^{T} R^{-1} B\right)+L_{4}^{N} .
$$

Let $E, D$ be as in (3.6). According to Lemma $5, L_{3}^{N}=f_{N-1}, L_{4}^{N}=-f_{N-2} E$, and $f_{n} D-f_{n-1} E=f_{n+1}$. Moreover, we have that

$$
\begin{aligned}
\nabla_{\boldsymbol{x}_{0}} \boldsymbol{x}_{N} & =f_{N-1} D-f_{N-2} E+f_{N-1}\left(Q A^{-T} Q_{B}^{-1}-Q A^{-T} Q\right) \\
& =f_{N}+f_{N-1}\left(Q A^{-T} Q_{B}^{-1}-Q A^{-T} Q^{-1}\right) .
\end{aligned}
$$

In turn, this leads to the inequality

$$
\left\|\nabla_{\boldsymbol{x}_{0}} \boldsymbol{x}_{N}\right\| \leq\left\|f_{N}-f_{N-1} Q A^{-T} Q^{-1}\right\|+\left\|f_{N-1}\right\|\left\|Q A^{-T} Q_{B}^{-1}\right\| .
$$

From Lemma 7 [ii] the first term is bounded, whereas the second term can be written as $\frac{f_{N-1}}{N}\left\|N Q A^{-T} Q_{B}^{-1}\right\|$, of which the first factor is bounded from Lemma $7[\mathrm{i}]$ and the second factor is $o(h)$ from Assumption [A]. Consequently $\left\|\nabla_{\boldsymbol{x}_{0}} \boldsymbol{x}_{N}\right\|$ is bounded, which proves the claim.

This result proves that even as $N \rightarrow \infty$, the essential components of our algorithm will stay bounded. In that regime, as our algorithm stores $\mathcal{O}(1)$ vectors, our storage will be $\mathcal{O}(1 / N)$ relative to a classical approach which stores all vectors $x_{i}$, a large and increasing storing efficiency.

4. Effect of numerical error. The computational core of our method is the recurrence equation (2.11). While Theorem 5 elucidates the stability effects in the linear case for the situation where that equation is computed exactly, an important practical issue is the effect of numerical error when evaluating (2.11). For moderatesized systems, all linear solves in that equation can be carried out directly, and that error is primarily of finite arithmetic type. For larger systems, some of the linear solves, particularly involving the matrix $\nabla_{x_{i}} \mathcal{M}\left(x_{i}\right)^{-T}$, may have to be carried out iteratively and, thus, will be inexact. This will yield another source of error that at most times is larger than the finite arithmetic one. The question thus becomes, How sensitive is this iteration to numerical error?

To have a comparable reference case, we compare the effect of numerical error against that for the forward equation (2.29d) in the strongly constrained $4 \mathrm{D}$-Var case, 
to which (2.11) is to a large extent analogous in this case. If one uses implicit methods to compute $x_{i}=M_{i}\left(x_{i}\right)$ in the strongly constrained case, then one of the components of the error is likely to be in the solution of linear equations with matrices derived from $\nabla_{x_{i}} \mathcal{M}\left(x_{i}\right)^{-T}$, so the errors would have a comparable source. As models of the error, we use

$$
\mathbf{e}_{i+1}^{s}=A \mathbf{e}_{i}^{s}+\epsilon_{i+1}^{s}, \quad i=0,1,2, \ldots, N-1,
$$

for the strong case and

$$
\tilde{\mathbf{e}}_{i+1}^{w}=\left(\begin{array}{c}
\mathbf{e}_{i+1}^{w} \\
\mathbf{e}_{i}^{w}
\end{array}\right)=L\left(\begin{array}{c}
\mathbf{e}_{i}^{w} \\
\mathbf{e}_{i-1}^{w}
\end{array}\right)+\tilde{\epsilon}_{i+1}^{w}=L \tilde{\mathbf{e}}_{i}^{w}+\tilde{\epsilon}_{i+1}^{s}, \quad i=1,2, \ldots, N-1,
$$

for the weak $4 \mathrm{D}$-Var case. In the weak case, $\mathbf{e}_{i}^{w}$ is the error in the state variable $x_{i}$, but for algebraic simplicity we focus on the joined successive states error $\tilde{\mathbf{e}}_{i}^{w}$; the two can easily be transformed into each other. Also, $\tilde{\epsilon}_{i}^{s}$ and, respectively, $\tilde{\epsilon}_{i}^{w}$ are the errors in evaluating the right-hand side of the recursion equations - the sources of error in this analysis.

These model equations one would obtain from (2.29d) and, respectively, (3.5)the latter derived from (2.11) - assuming a linear system of equations such as (3.1) and then subtracting the exact solution from the one affected by numerical error. From these models, we obtain

$$
\mathbf{e}_{N}^{s}=\sum_{n=0}^{N} A^{n} \epsilon_{N-n}^{s} \rightarrow\left\|\mathbf{e}_{N}^{s}\right\| \leq \sum_{n=0}^{N}\|A\|^{n}\left\|\epsilon_{N-n}^{s}\right\|
$$

for the strong case, where $\epsilon_{0}^{s}$ is the error in the initial condition. Here we use the triangle inequality and properties of matrix norms. Similarly, one obtains

$$
\tilde{\mathbf{e}}_{N}^{w}=\sum_{n=0}^{N-1} L^{n} \epsilon_{N-n}^{w} \rightarrow\left\|\tilde{\mathbf{e}}_{N}^{w}\right\| \leq \sum_{n=0}^{N-1}\|L\|^{n}\left\|\epsilon_{N-n}^{w}\right\|,
$$

where $\epsilon_{0}^{w}$ is the error from the initial iterate.

Under Assumption $[\mathrm{A}]$, we have that $A \sim I+O(h)$. Moreover, this is the case from the classical stability analysis where the standard assumption is that $A$ is diagonalizable [2]. Under this assumption, it follows that $A^{n}$ is bounded. On the other hand, under Assumption $[\mathrm{A}]$, the matrix $L,(3.6)$, with entries identified in Lemma 7 will tend in the limit to the matrix $\left[2 I_{s},-I_{s} ; I_{s}, 0\right]$ that has nontrivial $2 \times 2$ Jordan blocks. Therefore, as $n \rightarrow \infty$, it follows that $L^{n} \rightarrow \infty$. This is the main reason that the analysis from section 3 is so intricate. On the other hand, $L^{n}$ does not grow to $\infty$ faster than linearly, as follows from Lemma 7 .

1. If we have that $\|\epsilon\|_{i}^{s}$ and $\left\|\tilde{\epsilon}_{i}^{w}\right\|$ are bounded below, then the upper bounds on both $e_{N}^{w}$ in (4.3) and $e_{N}^{s}$ in (4.4) are going to $\infty$ as $N \rightarrow \infty$. Such is the case when the source of error cannot be reduced by the user, as is the case with truncation error.

2. If we can control the tolerance, then $\|\epsilon\|_{i}^{s} \leq \frac{1}{N}$ is sufficient to ensure that the error in (4.3) stays bounded in the strong case. The weak case, however, requires $\|\tilde{\epsilon}\|_{i}^{w} \leq \frac{1}{N^{2}}$ under the same considerations.

A conclusion for upper bounds is weaker than consequences for errors themselves, though this is a fairly standard practice in numerical analysis, and in the absence of further structure assumptions, these bounds are quite indicative of the behaviors of 
the numerical methods [2]. Moreover, results for accuracy of the gradient may be of interest; however, noting the expression of the functional (2.1), we see that upper bounds on errors in $x$ naturally extend to upper bounds on $\Gamma$ and its gradients using Lipschitz-type inequalities, so the conclusions would be largely similar.

We conclude that, under most scenarios, since $L^{N}$ is unbounded and $A^{N}$ is bounded, the recurrence we proposed for weak $4 \mathrm{D}$-Var $(2.11)$ will require more accuracy than the recurrence of the state in the strongly constrained case $(2.29 \mathrm{~d})$ for the accumulation of error to not overwhelm the calculation. We note, however, that (1) both recurrences must have to this end an error in their evaluation that goes to 0 as $N \rightarrow \infty$ (although (2.11) requires on the order of $N$ faster convergence to 0 ), and (2) in low-memory environments we are not aware of any alternatives for weak $4 \mathrm{D}$-Var to the recursive approached we propose here.

5. Numerical experiments. We now present numerical experiments that illustrate the theoretical findings discussed in section 2 . We solve both the nonlinear formulation (2.25), which we expect to be regular based on Theorem 3, and the optimization formulation with the objective (2.12), where a descent direction is obtained based on Theorem 4. To solve the nonlinear equation (2.25) in a low-memory fashion we use the L-Broyden method defined in [27], whereas for the optimization approach we use L-BFGS [15]. Such methods used a fixed number of stored vectors $p$, which will be specified in numerical experiments. Moreover, we perform a comparison between the low-memory approach and the weakly constrained 4D-Var.

5.1. Model problem. In this study we focus on Burgers' equation (see $[1,11$, $26,19]$ ), which describes the interaction between nonlinear advection and turbulent dissipation. This equation is a fundamental problem in fluid mechanics and has been used extensively as a benchmark in meteorology (see [11] and references therein). The inviscid form $(\mu=0)$ is also important because it captures the essence of the large-scale transient waves of mid-latitudes. Variational data assimilation for Burgers' equation is discussed in [1].

Burgers' equation has the following definition:

$$
\begin{aligned}
& \frac{\partial u}{\partial t}+\frac{1}{2} \frac{\partial\left(u^{2}\right)}{\partial x}=\mu \frac{\partial^{2} u}{\partial x^{2}}, \quad x \in(0,1) \times(0, T), \quad \mu>0 \\
& u(0, t)=u(1, t)=0 \\
& u(x, 0)=u_{0}(x)
\end{aligned}
$$

Here $\mu$ is the viscosity coefficient. The solution of Burgers' equation with viscosity coefficient $\mu=0.01,0.1,0.5,1$ is shown in Figure 5.1.

As seen in Figure 5.1, the function value drops sharply when the viscosity coefficient is larger than 0.5. In such cases, the information content is limited, and therefore, we choose the cases when $\mu$ is small.

In terms of the numerical discretization of the problem, we let $u_{j}^{m}$ denote the function value $u(j \triangle x, m \triangle t)$. According to [1], a centered finite-difference scheme for Burgers' equation is

$$
\frac{u_{j}^{m+1}-u_{j}^{m}}{\triangle t}+\frac{\left(u_{j+1}^{m}\right)^{2}-\left(u_{j-1}^{m}\right)^{2}}{4 \triangle x}-\frac{\mu}{(\triangle x)^{2}}\left(u_{j+1}^{m+1}-2 u_{j}^{m+1}+u_{j-1}^{m+1}\right)=0 .
$$

Let $U^{m}$ denote the vector determined by $u_{j}^{m}, j=0, \ldots, N$. The scheme in (5.2) results in a discrete dynamical system that can be written compactly as $P U^{m+1}=$ $\mathcal{S}\left(U^{m}\right)$. Here, $P$ is a symmetric tridiagonal matrix with $\left(1+2 \mu(\triangle t) /(\triangle x)^{2}\right)$ on 


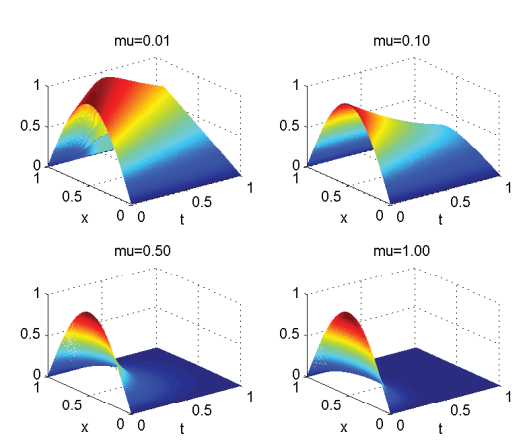

FIG. 5.1. Burgers' equation with viscosity coefficient $\mu=0.01,0.1,0.5,1$ with initial condition $u(x, 0)=\sin (\pi x)$.

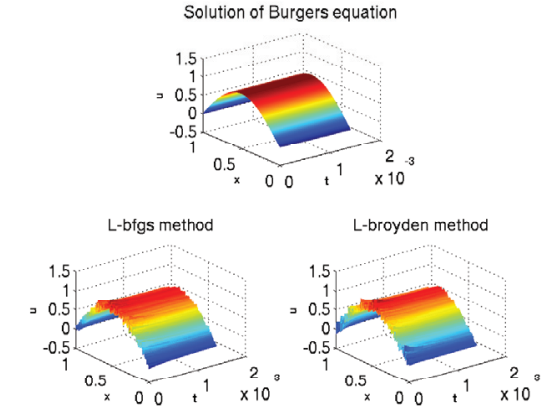

FIG. 5.2. Numerical solutions for lowmemory implementation with L-BFGS (bottom left) and L-Broyden (bottom right) methods and the solution of the Burgers' equation (top) for $\mu=0.01, \Delta t=\Delta x / 1000$, and $N=700$.

the diagonal and $-\mu(\triangle t) /(\triangle x)^{2}$ on the sub- and superdiagonals. This defines the discrete dynamical mapping $\mathcal{M}(\cdot)$ discussed in section 2 . Specifically, we have that $\mathcal{M}_{i}\left(U^{i}\right)=P^{-1} \mathcal{S}\left(U^{i}\right)$ and $\nabla \mathcal{M}_{i}\left(U^{i}\right)=P^{-1} \nabla \mathcal{S}\left(U^{i}\right)$. Obviously, $P=I+\frac{T}{N} B^{0}, B^{0}$ is a tridiagonal matrix, with $\frac{2 \mu}{(\triangle x)^{2}}$ on the diagonal and $-\frac{\mu}{(\Delta x)^{2}}$ above and below, and $\nabla \mathcal{S}\left(U^{i}\right)=I-\frac{T}{N}\left(B^{1}\right)$, where $B^{1}$ is the tridiagonal matrix with zero on the diagonal, $\frac{U_{2: N}^{i}}{2 \triangle x}$ on the superdiagonal, and $-\frac{U_{1: N-1}^{i}}{2 \triangle x}$ on the subdiagonal. Hence $\nabla \mathcal{M}_{i}\left(U^{i}\right)=$ $I+\frac{T}{N} B^{3}+\cdots$ with $B^{3}=B^{1}-B^{0}$. Therefore $\mathcal{M}(\cdot)$ satisfies all the conditions required of it for the theoretical developments in section 2 .

However, not every finite-difference scheme has this property. A counterexample is the implicit Lax-Friedrichs scheme discussed by [1]. This scheme uses the average of $u_{j+1}^{m}$ and $u_{j-1}^{m}$ in place of $u_{j}^{i}$. Doing so leads to $\nabla \mathcal{M}_{i}\left(U^{i}\right)$ violating Assumption [A].

5.2. Numerical results. We now describe in detail the numerical experiments, the objective being the minimization of (2.12). The function $\mathcal{M}_{i}$ in (2.2) is derived from the centered finite-difference scheme applied to Burgers' equation. We consider $\triangle x=1 / 501$ and $U^{0}=\sin (\pi x)$ to generate the data set $\mathcal{G}:=\left\{U^{0}, \mathcal{M}_{i}\left(U^{i}\right), i=\right.$ $1, \ldots, N\}$. We use $x_{B}=U^{0}$ as the background vector. The observation data are computed by applying $\mathcal{H}_{i}(\mathcal{G})$ and perturbed by normal random noise times with standard deviation 0.1 to mimic the action of a noisy nonlinear operator. In particular, we select $\mathcal{H}(\circ)=\sin (\circ)$. To be closer to a real situation, the observations are taken every 10 steps in space-time (i.e., at time node $i 10 \triangle t$ and space node $i 10 \triangle x$ ).

We use the L-BFGS algorithm to compute the minimizer of (2.12) (but with search direction $\Theta_{N}$ as indicated by Theorem 4). We also use the L-Broyden algorithm to compute the solution of (2.25). We choose $Q$ to be a diagonal matrix $(\triangle t)^{2}[2,1, \ldots, 1,2]$ on the diagonal and $Q_{B}^{-1}$ and $R^{-1}$ to be $100 I$. The initial solution $U^{0}$ is perturbed with normal random noise times with standard deviation 0.1 and used as the initial guess for this algorithm. Note that only the $y$-axis of each plot of results is set to be $\log$ scaled. Also note that all numerical results are scaled by the corresponding values of the initial guess.

5.2.1. Results for Burgers' equation. In Figures 5.3 and 5.4, we plot function values of $\widehat{\Gamma}$ as in (2.12) at each iteration of the L-BFGS algorithm. We compare the 


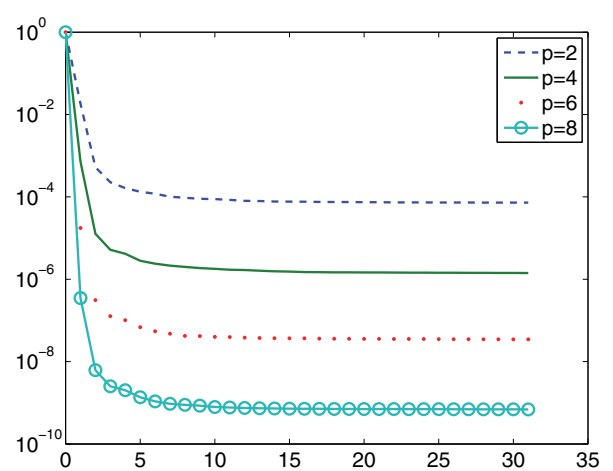

FIG. 5.3. Scaled function value of (2.12) at each iteration of $L$-BFGS for $\mu=0.01, \triangle t=$ $\triangle x / 1000, N=700$, and $p=2,4,6,8$ stored vectors.

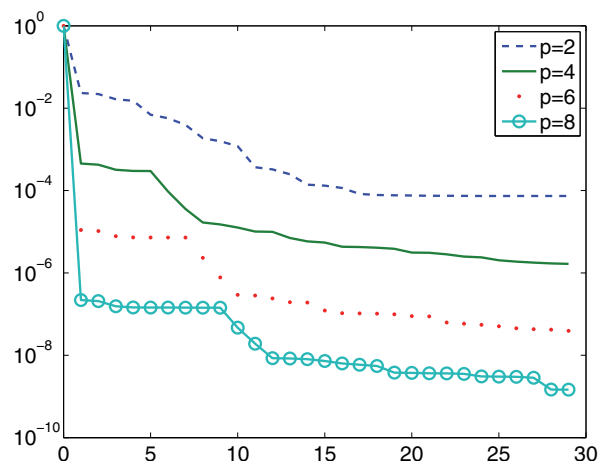

FIG. 5.5. Scaled function values of $\widehat{\Gamma}$ as in (2.12) at each iteration of $L$-Broyden algorithm for $p=2,4,6,8$, stored vectors, $\mu=0.01, \Delta t=$ $\triangle x / 1000$, and $N=700$.

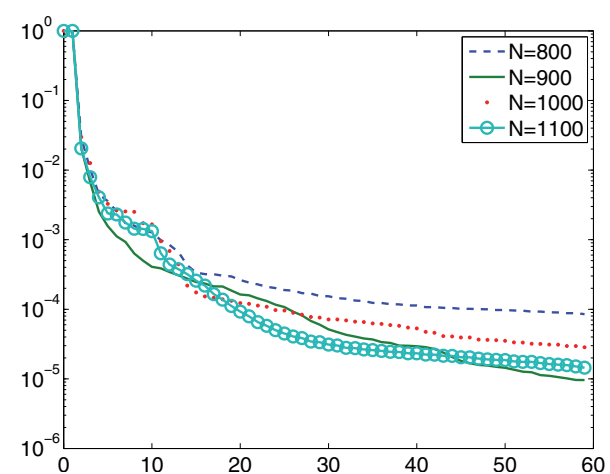

FIG. 5.4. Scaled function value of (2.12) at each iteration of L-BFGS for $\mu=0.01$, $\triangle t=\triangle x / 1000, p=6$ stored vectors, and $N=800,900,1000,1100$.

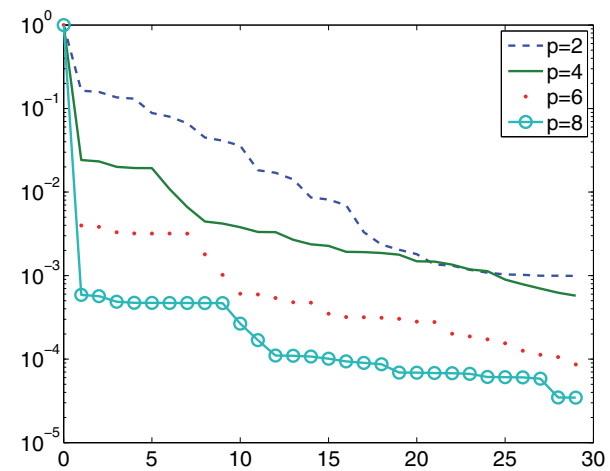

FIG. 5.6. Scaled norms of residuals of (2.26) at each iteration of $L$-Broyden algorithm for $p=2,4,6,8$, stored vectors, $\mu=0.01$, $\triangle t=\triangle x / 1000$, and $N=700$.

results obtained by using different numbers of stored vectors $p=2,4,6,8$ for $N=700$ in Figure 5.3. Note that the convergence rates depend highly on the number of stored vectors, $p$. In Figure 5.4, we plot the results of $N=800,900,1000,1100$. In Figures 5.5 and 5.6, we plot function values of (2.12) at each iteration of the L-Broyden algorithm. In Figures 5.7 and 5.8 we show the norms of residuals of (2.26) at each iteration of the L-Broyden algorithm. In Figures 5.5 and 5.6 we compare the results obtained when using the L-Broyden method for different numbers of stored vectors $p=2,4,6,8$ when $N=700$, where we see again the same dependence on $p$. The results for L-Broyden with $N=800,900,1000,1100$ for $p=4$ are shown in Figures 5.7 and 5.8. We see from our numerical simulations that the objective function is significantly reduced (by 2-5 orders of magnitude).

Though the problems are not solved to high accuracy, the solution does approach a perturbed version of the original solution, as can be seen in Figures 5.2, 5.9, and 5.10. There, we illustrate the numerical solutions of L-BFGS (Figure 5.2, bottom left) and L-Broyden (Figure 5.2, bottom right) methods together with the solution of the 


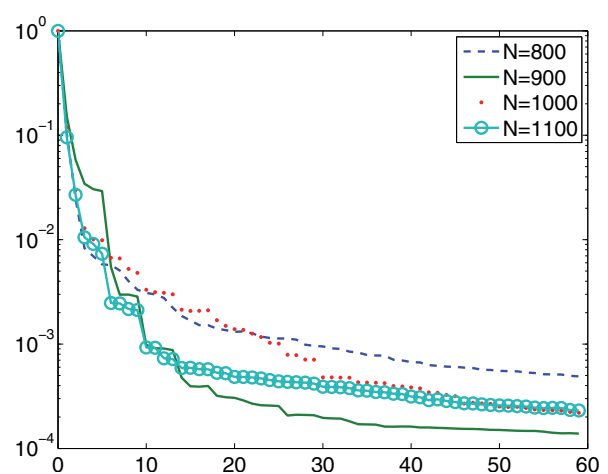

FIG. 5.7. Scaled function values of $\widehat{\Gamma}$ as in (2.12) at each iteration of $L$-Broyden algorithm for $N=800,900,1000,1100, p=4$ stored vectors, $\mu=0.01$, and $\triangle t=\triangle x / 1000$.
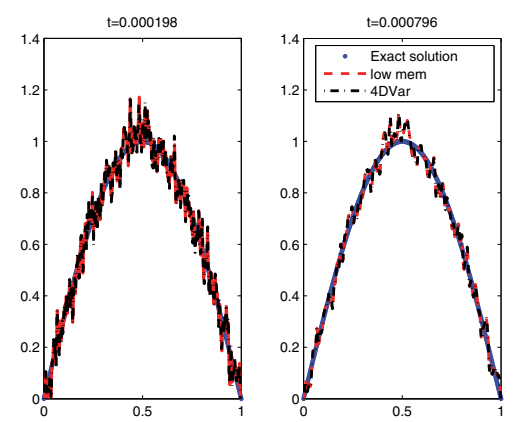

FIG. 5.9. Numerical solutions of the lowmemory implementation and $4 D$-Var methods and the solution of the Burgers' equation at fixed time nodes for $\mu=0.01, \Delta t=\Delta x / 1000$, and $N=700$.

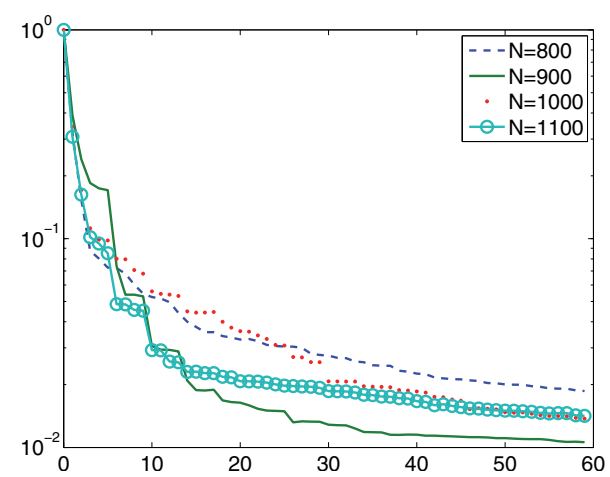

FIG. 5.8. Scaled norms of residuals of (2.26) at each iteration of $L$-Broyden algorithm for $N=800,900,1000,1100, p=4$ stored vectors, $\mu=0.01$, and $\triangle t=\triangle x / 1000$.
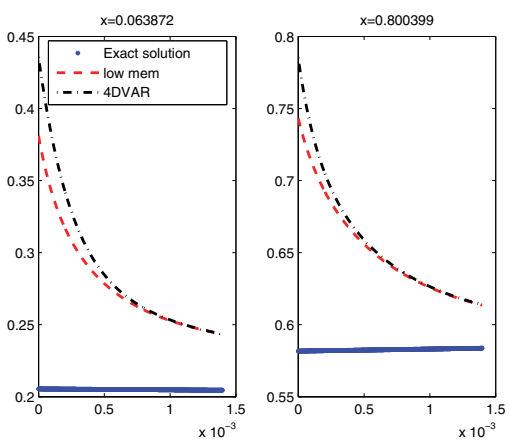

FIG. 5.10. Numerical solutions of the lowmemory implementation and 4D-Var methods and the solution of the Burgers' equation at fixed space nodes for $\mu=0.01, \Delta t=\Delta x / 1000$, and $N=700$.

Burgers' equation (Figure 5.2, top) for $\mu=0.01, \triangle t=\triangle x / 1000$, and $N=700$. We conclude that the findings of Theorem 3 are valid in this case.

Certainly, this is a limited set of experiments, for example, $\Delta t$ is much smaller than would be used in practical problems, and for large $\Delta t$ we have definitely seen the instability that we analyze in section 3 and which we can guarantee to not occur only for fixed $T$ and $\Delta t$ sufficiently small. Also, we do not find in the experiments a large dependence with $p$ which is uncommon for quasi-Newton methods, which also indicates that the circumstances here are quite particular.

Nevertheless, in these limited circumstances (which are the only ones in which we can guarantee at the moment that the method works for large and increasing $N$, where the method would be practically interesting) we observe that $p$ can stay essentially $\mathcal{O}(1)$ and still achieve convergence. We can see from the results described above that the L-BFGS method using only forward sweeps converges faster than L-Broyden, though our theory here through Theorem 4 applies only in the regime of small $T$. Overall, we find that the numerical experiments validate the findings expressed in Theorems 3 and 4 from section 2, that the nonlinear equation obtained 


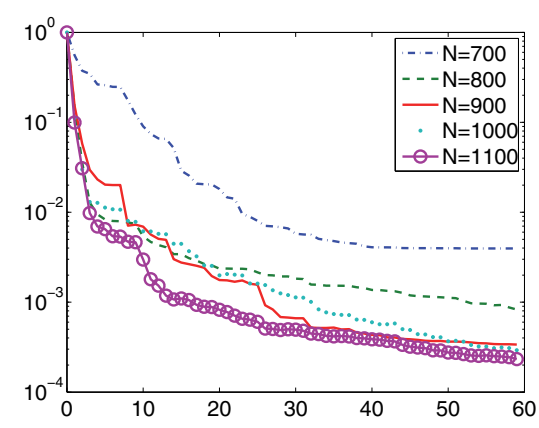

FIG. 5.11. Scaled function values of $\widehat{\Gamma}$ as in (2.12) at each iteration of $L$-Broyden algorithm for modified Burgers' equation for $p=6$ stored vectors, $\mu=0.01$, and $\triangle t=\triangle x / 1000$

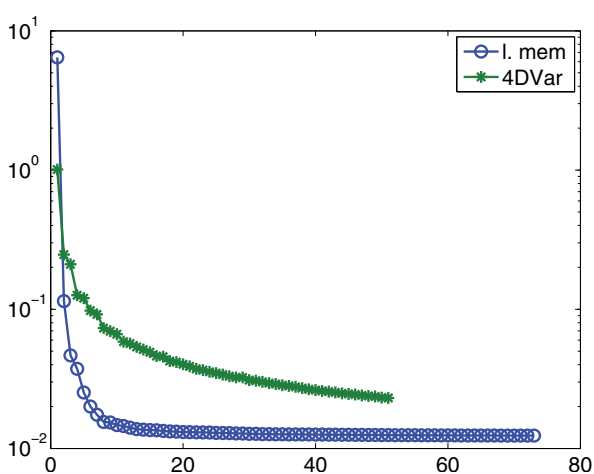

FIG. 5.12. Comparison of function values between 4D-Var method and reduced-memory method for $N=700, p=6, \mu=0.01$, and $\triangle t=\triangle x / 1000$.

by our reduction procedure (2.12) is well-posed and can be solved both by using the L-Broyden method or L-BFGS method with forward sweeps only even though $p$ is much smaller than the dimension of $x$.

5.2.2. Augmented Burgers' equation. In order to replicate effects found in higher-dimensional geophysical problems we consider adding a skew-symmetric term that accounts for rotational effects. To this end, we augment the Burgers' equation (5.1) with $\kappa \partial u / \partial x$ :

$$
\frac{\partial u}{\partial t}+\frac{1}{2} \frac{\partial\left(u^{2}\right)}{\partial x}=\left(\mu \frac{\partial^{2}}{\partial x^{2}}+\kappa \frac{\partial}{\partial x}\right) u .
$$

If discretized with central differences, the constant advective term becomes skewsymmetric. In Figure 5.11, we illustrate the function values of the cost function (2.12) at each iteration of the L-Broyden algorithm with $p=6$ for the modified Burgers' equation with $\kappa=0.1$. We observe the same convergence behavior as in the case of the Burgers' equation in section 5.2.1. We conclude that the findings of Theorem 3 are valid in this case as well.

5.2.3. Linear PDE. For completeness we also include results with a simple linear PDE (linear advection),

$$
\begin{aligned}
\frac{\partial u}{\partial t} & =\mu \frac{\partial u}{\partial x}, \quad x \in(0,1) \times(0, T), \quad \mu>0, \\
u(0, t) & =u(1, t)=0, \\
u(x, 0) & =\sin (\pi x),
\end{aligned}
$$

using the same setup as above. The results showing a fast convergence are illustrated in Figure 5.13. We conclude that the findings of Theorem 4 are valid in this case as well.

5.2.4. Comparison between reduced-memory and weakly constrained 4D-Var. In Figure 5.12, we show the cost function values of (2.12) and that of (2.1) using the 4D-Var algorithm at each iteration of the L-BFGS algorithm with $p=6$ stored vectors at $N=700$. We note that the search space for the reducedmemory algorithm is $N$-fold smaller than in the $4 \mathrm{D}$-Var case, and therefore, the 


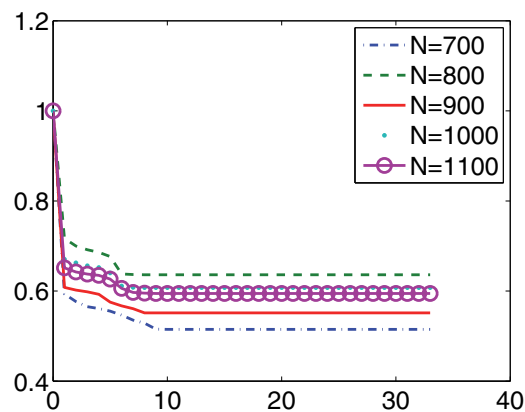

Scaled function value of (2.12).

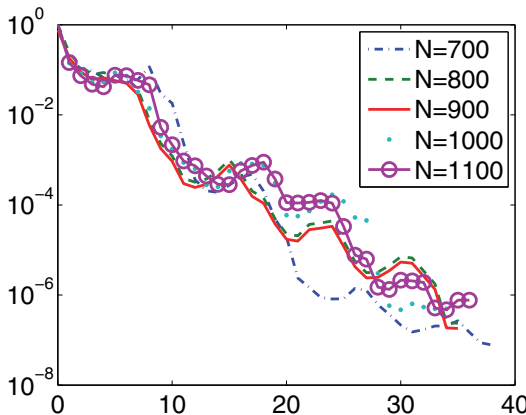

Norm of descent direction.

FIG. 5.13. Scaled objective function value and gradient vs. iteration number results for (5.4) with $\Delta t=\Delta x / 1000$, using the L-BFGS method with $p=6$ stored vectors.

reduced-memory algorithm may converge faster than the weakly constrained 4D-Var algorithm. We compare quasi-Newton methods on the reduced problem with quasiNewton methods for weakly constrained 4D-Var using the same number of stored vectors $p$. As the latter problem has a state space that is $N$ times larger, where $N$ is the number of time steps considered, it follows that our algorithm uses $N$ times less memory. We thus achieved significant memory savings: as we have solved problems with $N=700$, the reduction in memory usage is also a factor of 700 . In Figures 5.9 and 5.10 we also compare the quality of the predictions of the two methods and we note that the results are similar.

We conclude that our theoretical findings are valid and that the method proposed in this study has the potential for large memory savings as well as faster convergence.

5.2.5. Exploring wider parameter ranges. We next consider two cases that have parameter ranges closer to those of the intended application target. In the first case we investigate the effects of larger model errors on the performance of our approach. Here, we reduce the number of observations that are now taken every 11 steps in time (i.e., at time node $i 11 \Delta t$ ) and every 100 steps in space (i.e., at space node $i 100 \Delta x$ ) and use covariance matrices $Q=R=0.001 I, Q_{b}=0.01 I$, with $\Delta t=\Delta x / 1000, \Delta x=1 / 500$, and $N=110$. The resulting cost function decrease is shown in Figure 5.14(a).

In the second case we investigate the effects of larger observation time intervals. To this end, we increase the time step length to $\Delta t=1 / 23,834, \Delta x=1 / 700$; the observations are taken every 31 steps in time (i.e., at $30 i \Delta t=1 / 768$ ) and every 200 steps in space (i.e., at $i 200 \Delta x$ ). The resulting observation time interval is larger but comparable to the decaying time of the smallest scale processes as estimated by a Fourier analysis. The total observation time interval is $N=32$. We selected $Q=10^{-8} I, R=10^{-2} I, Q_{b}=10^{-3} I$, and $p=6$. The result is shown in Figure 5.14(b).

In both cases we used an L-BFGS optimization algorithm around our reduced low-memory approach and we compare it with the L-BFGS approach applied to the weakly constrained 4D-Var problem. For $N$ larger than described above, our approach does cause difficulties. In particular, negative rank-one BFGS updates are obtained and the standard definition of L-BFGS methods [15, section 7.2] breaks down in these situations. While exploring more robust versions of L-BFGS approaches is an important aim for future research, we also note that the results in Theorems 3 and 4 


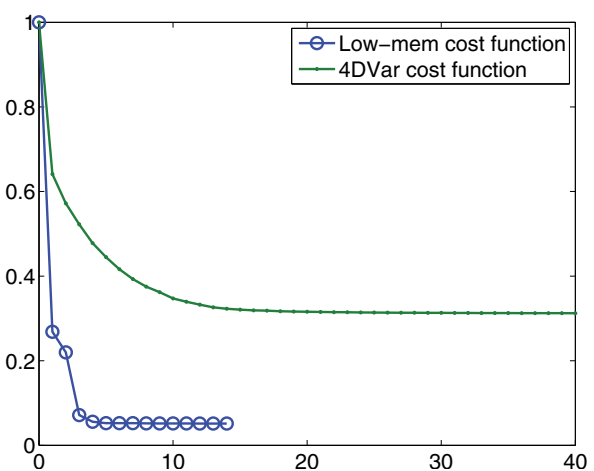

(a) $\Delta t=1 / 500,000$.

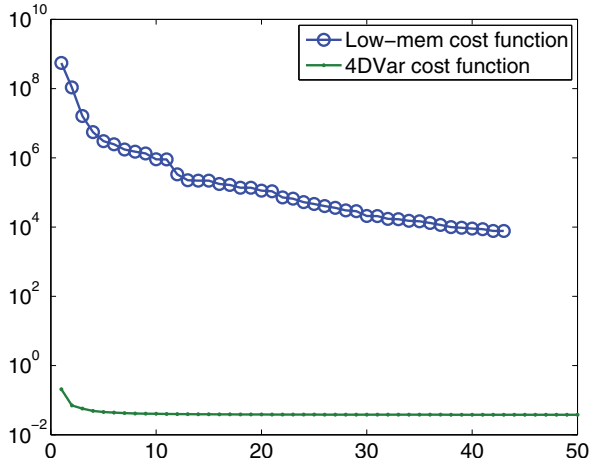

(b) $\Delta t=1 / 23,834$.

FIG. 5.14. Comparison between low-memory and weak-constrained $4 D$-Var when using $Q=$ $R=10^{-3} I, Q_{b}=10^{-2} I$, (a) $\Delta t=1 / 500,000, p=6, N=110$, and (b) $\Delta t=1 / 23,834$ and observation gap in time of $1 / 768 Q=10^{-8} I, R=10^{-2} I, Q_{b}=10^{-3} I, p=6, N=32$.

are local in nature, and the result in Theorem 4 also requires a sufficiently small horizon, and therefore they do not preclude such an outcome away from the solution. In both cases, the memory savings, though smaller than in section 5.2.4, are still significant: since $N=110$ in the first case and $N=32$ in the second, so are the memory reduction factors.

In the second case, the L-BFGS is terminated at iteration 43 due to encountering a negative BFGS update; however, it made good progress up to that point. In addition, the problem setup is at the stability limit of the method: increasing the error matrix $Q$ or the time step $\Delta t$ beyond our choices makes the computation fail due to rapid divergence of the recursion. This is also indicated by the large initial value of the objective function. This is not entirely unexpected from the discussion in section 3 . In the end, this suggests that the method works for $\Delta t$ small enough, which for practical applications also means an observation time interval small enough.

We conclude that in these cases where we increase the model error level and the observational time interval the memory savings can still be significant; however, this finding is mitigated by the fact that several technical difficulties do appear such as negative updates for L-BFGS and instability. Eventually, it seems clear from these experiments that with the method at this stage of development, increasing the model error level or the observational time interval will result in numerical difficulties. This is particularly true in the second case illustrated above, where in practice the observation time interval is comparable to the decaying time of the smallest scale processes. We anticipate that these issues can be alleviated with robust L-BFGS implementations and multiple shooting ideas that trade memory for stability. This will be pursued in future studies.

6. Conclusions. Hidden Markov models with physical model error pose new challenges to data assimilation. One of these challenges is the fact that, being weakly constrained, the model can no longer be used to reduce the storage needs by deriving one state from another state. Instead, the entire estimated trajectory must be stored. This challenge is particularly burdensome with the emergence of new architectures where less memory will be available per node.

We addressed this challenge by using a new approach, which constrains the problem with the optimality conditions at the states other than initial. In turn, this results 
in a nonlinear equation whose residual vector can be computed by forward sweeps only, or an optimization problem where an approximation to the gradient can be computed with forward sweeps only. In turn, no intermediate states need to be stored for advancing the best estimate algorithm. In conjunction with limited-memory algorithms (Broyden or BFGS) we can solve such a problem with low or even $\mathcal{O}(1)$ storage. We validated these findings in the low model error and small observation time interval regime on numerical experiments with Burgers' equations, augmented Burgers' equation, and a linear advection problem. Moreover, we carried out a comparison of the L-BFGS version of our approach with L-BFGS applied to the full weakly constrained 4D-Var experiment using Burgers' equation, and memory savings with a factor of 700 were obtained.

On the other hand, the approach poses other issues; in particular, it is prone to instability problems; for example, a simplified error analysis indicates that our recurrence requires more accuracy in its evaluation than the strongly constrained $4 \mathrm{D}$-Var forward sweep. Our proofs in the interesting case - that of a large number of time steps $N$-work only in the limit of small time step $\Delta t$, and the numerical demonstrations are also done in this regime. We have also investigated numerical cases with larger model errors and larger observation time intervals, the latter on the order of the decaying time of the smallest scale processes. In both cases, the memory savings, though smaller than in the previous examples, are still significant: reduction factors of 110 were obtained for one case and 32 for the other. In both cases, larger $N$ resulted in negative updates for the L-BFGS methods applied to the reduced problems. The second case was also interrupted by negative updates after good progress, and increasing $\Delta t$ and the model error beyond our choices displayed instability of the recursion. We conclude that a larger model error and larger time steps pose challenges to our approach and require further investigation.

To bring the method closer to a practical regime for its target applications, we will pursue several other avenues such as more robust versions of L-BFGS methods, multiple shooting to address stability concerns, and preconditioning based on a coarser or reduced system. Nevertheless, we believe that algorithms reducing storage (and implicitly, communication) are important issues that this method helps to address and for which few other options seem to exist.

\section{Appendix A. Proofs of lemmas in section 3.}

A.1. Proof of Lemma 3. Let $A=I+h P_{1}$, with $P_{1}:=P_{1}(h)=P+\mathcal{O}(h)$. For $h$ sufficiently small the series expansion of $\left(I+h P_{1}\right)$ in $h$ holds to give

$$
\begin{aligned}
& \left\|Q A^{-T} Q^{-1}\right\|^{N}=\left\|\sum_{i=0}^{\infty}\left(-h Q P_{1}^{T} Q^{-1}\right)^{i}\right\|^{N} \\
& \leq\left(\sum_{i=0}^{\infty}\left(h\|Q\|\left\|P_{1}^{T}\right\|\left\|Q^{-1}\right\|\right)^{i}\right)^{N}=\left(1-h\|Q\|\left\|P_{1}^{T}\right\|\left\|Q^{-1}\right\|\right)^{-N} .
\end{aligned}
$$

When $N \rightarrow \infty,\left(1-h\|Q\|\left\|Q^{-1}\right\|\left\|P_{1}^{T}\right\|\right)^{-N} \rightarrow \exp \left(T\left\|Q_{0}\right\|\left\|Q_{0}^{-1}\right\|\left\|P^{T}\right\|\right)$. Similarly,

$$
\left\|Q A^{T} Q^{-1}\right\|^{N} \leq\left\|I+h Q P_{1}^{T} Q^{-1}\right\|^{N} \leq\left(1+h\|Q\|\left\|Q^{-1}\right\|\left\|P_{1}^{T}\right\|\right)^{N}
$$

and $\|A\|^{N}=\left\|I+h P_{1}\right\|^{N} \leq\left(1+h\left\|P_{1}\right\|\right)^{N}$. When $N \rightarrow \infty$, we have that

$$
\left(1+h\|Q\|\left\|Q^{-1}\right\|\left\|P_{1}^{T}\right\|\right)^{N} \rightarrow \exp \left(T\left\|Q_{0}\right\|\left\|Q_{0}^{-1}\right\|\left\|P^{T}\right\|\right)
$$


and $\left(1+h\left\|P_{1}\right\|\right)^{N} \rightarrow \exp (T\|P\|)$. Hence the boundedness of the quantities in the statement follow since sequences admitting limits are bounded.

A.2. Proof of Lemma 4. It is easy to verify that (3.7) holds for $L^{1}$ because $g_{0}=I_{s}, U+V=g_{1},-U V=-g_{0} U V$, and $g_{1}-g_{0}(U+V)=0$. Assume that (3.7) holds for $L^{n}$ :

$$
\begin{aligned}
L^{n+1} & =\left(\begin{array}{cc}
g_{n} & -g_{n-1} U V \\
g_{n-1} & g_{n}-g_{n-1}(U+V)
\end{array}\right)\left(\begin{array}{cc}
U+V & -U V \\
I_{s} & 0
\end{array}\right) \\
& =\left(\begin{array}{cc}
g_{n}(U+V)-g_{n-1} U V & -g_{n} U V \\
g_{n} & -g_{n-1} U V
\end{array}\right) .
\end{aligned}
$$

We also have that

$$
\begin{aligned}
g_{n}(U+V)-g_{n-1} U V & =\sum_{i=0}^{n} V^{i} U^{n-i} U+\sum_{i=0}^{n} V^{i} U^{n-i} V-\sum_{i=0}^{n-1} V^{i} U^{n-1-i} U V \\
& =\sum_{i=0}^{n} V^{i} U^{n+1-i}+\sum_{i=0}^{n} V^{i} U^{n-i} V-\sum_{i=0}^{n-1} V^{i} U^{n-i} V \\
& =\sum_{i=0}^{n+1} V^{i} U^{n+1-i}=g_{n+1} .
\end{aligned}
$$

This proves the induction hypothesis for the upper left corner element of $L^{n+1}$. By rearranging the above equality we obtain $g_{n+1}-g_{n}(U+V)=-g_{n-1} U V$, which demonstrates the induction hypothesis for the lower right element. Since the other elements of $L^{n+1}$ are in the algebraic form required by the induction hypothesis, the proof is complete.

A.3. Proof of Lemma 5. Let $f_{n}$ be defined by the above recursion and initial conditions. The case $n=1$ immediately holds, and the recursion relation can immediately be verified by inspection.

A.4. Proof of Lemma 6. We write (3.9) in an equivalent form by introducing the matrix-valued mappings $\Psi_{1}(h), \Psi_{2}(h)$ satisfying $\widehat{U}(h)=I_{s}+h J_{1}(h)+h \Psi_{1}(h)$ and $\widehat{V}(h)=I_{s}-h J_{2}(h)-h \Psi_{2}(h)$. It immediately follows that the first equation in (3.9) is equivalent to

$$
h\left(\Psi_{1}(h)-\Psi_{2}(h)\right)=C(h) .
$$

Replacing the same ansatz in the second equation of (3.9), we obtain that

$$
\begin{array}{r}
\left(I_{s}+h J_{1}(h)+h \Psi_{1}(h)\right)\left(I_{s}-h J_{2}(h)-h \Psi_{2}(h)\right)=\left(I_{s}+h J_{1}(h)\right)\left(I_{s}-h J_{2}(h)\right) \\
+h \Psi_{1}(h)\left(I_{s}-h J_{2}(h)\right)-\left(I_{s}+h J_{1}(h)\right) h \Psi_{2}(h)-h^{2} \Psi_{1}(h) \Psi_{2}(h)=0 .
\end{array}
$$

Replacing now $\Psi_{2}(h)$ from (A.2) in the last relationship, and dividing by $h^{2}$, we obtain that (3.9) holds if and only if there exists $\Psi=\Psi_{1}(h)$ such that

$$
\Theta(h ; \Psi):=-\Psi\left(J_{2}(h)+\frac{C(h)}{h}\right)-J_{1}(h) \Psi+\frac{C(h)}{h^{2}}+J_{1}(h) \frac{C(h)}{h}+\Psi^{2}=0 .
$$

By our assumptions, the mapping $\Theta(h ; \Psi)$ is continuous in $h$ and infinitely differentiable in $\Psi$ (in effect, polynomial), and so are all its derivatives with respect to $\Psi$. 
It also satisfies $\Theta\left(0, \mathbf{0}_{s}\right)=0$. The action of its $\Psi$ derivative at the point $\left(0, \mathbf{0}_{s}\right)$ along a direction $\Psi_{d}$ (which can be seen as a matrix in $\mathbb{R}^{s \times s}$, making the derivative a four-dimensional tensor) satisfies

$$
\nabla_{\Psi} \Theta\left(0, \mathbf{0}_{s}\right) \Psi_{d}=-\Psi_{d} J_{1}^{0}-J_{2}^{0} \Psi_{d}
$$

The right-hand side of (A.4) is closely connected to Sylvester's equation: $A X+X B=$ $C$, where $A, B, C \in \mathbb{R}^{s \times s}$ and $X$ is an unknown matrix in $\mathbb{R}^{s \times s}$. If $A$ and $-B$ have no common eigenvalues, then Sylvester's equation has a unique solution $X$ for every $C$ [23, Theorem 1.16]. As $J_{1}^{0}$ and $-J_{2}^{0}$ have no common eigenvalues, it follows from the properties of Sylvester's equation that the mapping $\nabla_{\Psi} \Theta\left(0, \mathbf{0}_{s}\right) \Psi_{d}$ is one-to-one and onto on $\mathbb{R}^{s \times s}$, and thus invertible with an inverse we denote by $\nabla_{\Psi} \Theta^{-1}$. This makes the equation (A.3), $\Theta(h, \Psi)=0$, regular at $\left(0, \mathbf{0}_{s}\right)$, and thus defines locally $\Psi$ uniquely as a function of $h$.

Since all the derivatives with $\Psi$ of $\Theta$ are continuous in $h$, it follows that there exists a neighborhood of $\left(0, \mathbf{0}_{s}\right)$ in which $\Theta, \nabla_{\Psi} \Theta$, and $\nabla_{\Psi}(\Theta)^{-1}$ exist and are continuous and their norms are bounded by $C_{\theta}$. Moreover, $\nabla_{\Psi} \Theta$ is uniformly Lipschitz in $\Psi$ with respect to $h$ (as it is differentiable, and its derivative is continuous in $h$ and $\Psi$ ). We assume without loss of generality that the Lipschitz constant is upper bounded by $C_{\theta}$.

We also have from (A.3) that $\Theta\left(h ; \mathbf{0}_{s}\right)=\frac{C}{h^{2}}+\frac{C}{h} \Psi_{1}(h)=\beta(h)$, with $\|\beta(h)\| \rightarrow 0$ as $h \rightarrow 0$. It then follow that there exists an $h_{0}$ such as $\alpha(h)=\eta(h) C_{\theta}^{2} \leq \frac{1}{2}$ for all $0 \leq h \leq h_{0}$, where

$$
\eta(h)=\left\|\nabla_{\Psi} \Theta\left(h, \mathbf{0}_{s}\right)^{-1} \Theta\left(h, \mathbf{0}_{s}\right)\right\| \leq C_{f}\|\beta(h)\| .
$$

As a result, the conditions for Kantorovich's theorem [17, section 12.6.2] are met. There exists a solution of the equation $\Theta\left(h, \Psi_{1}(h)\right)=0$ satisfying $\left\|\Psi_{1}(h)\right\| \leq C_{\Psi} \beta(h)$ for some $C_{\Psi}>0$ and all $h \leq h_{0}$.

From the equivalence of (A.3) with (3.9) it follows that $\widehat{U}(h)$ and $\widehat{V}(h)$ exist and satisfy $\widehat{U}(h)-U(h)=h \Psi_{1}(h)=o(h)$ and $\widehat{V}(h)-V(h)=h \Psi_{2}(h)=C-h \Psi_{1}(h)=o(h)$. This proves (3.10) and the claim.

A.5. Proof of Lemma 7. We first verify that the conditions needed to use Lemma 6 apply. With the definition of $U$ we have that $U(h)=Q(h) A^{-T}(h) Q(h)^{-1}=$ $I+h Q_{0} P^{T} Q_{0}^{-1}+\mathcal{O}\left(h^{2}\right), V(h)=A(h)=I+h P+\mathcal{O}\left(h^{2}\right)$, and $C(h)=o\left(h^{2}\right)$. Moreover, $Q_{0} P^{T} Q_{0}^{-1}$ has the same eigenvalues as $P^{T}$ and $P$. Therefore $Q_{0} P^{T} Q_{0}^{-1}$ has common eigenvalues with $-P$ if and only if $P^{T}$ and $-P$ do, which is excluded by our hypothesis.

Therefore the conclusions of Lemma 6 apply to give matrices $\widehat{U}$ and $\widehat{V}$ satisfying (3.9) and (3.10). It then follows that the matrix $L$ in (3.6) has the same form as in Lemma 4, and application of that result in conjunction with the definition of $f_{n}$ in Lemma 5 results in

$$
f_{n}=\sum_{i=0}^{n} \widehat{V}^{i} \widehat{U}^{n-i} .
$$

In turn, this implies that

$$
\frac{\left\|f_{n}\right\|}{N+1} \leq \max \left\{\|\widehat{V}\|^{N},\|\widehat{U}\|^{N}\right\}
$$

From the fact that $h=\frac{T}{N}$ and (3.10) it follows that $\|\widehat{V}\|^{N} \leq(1+h\|P\|+o(h))^{N} \rightarrow$ $\exp \{(\|P\| T)\}$, and the sequence is thus bounded. From similar arguments, so is $\|\widehat{V}\|$, which proves part [i] of the claim. 
For part [ii] we notice from (A.6) that

$$
f_{n}-f_{n-1} \widehat{U}=\sum_{i=0}^{n} \widehat{V}^{i} \widehat{U}^{n-i}-\sum_{i=0}^{n-1} \widehat{V}^{i} \widehat{U}^{n-i}=\widehat{V}^{n} .
$$

Using $Q A^{-T} Q^{-1}=\widehat{U}+o(h)$ from (3.10), we obtain that

$$
\begin{aligned}
\left\|f_{N}-f_{N-1} Q A^{-T} Q^{-1}-I_{s}\right\| & \leq\left\|f_{N}-f_{N-1} \widehat{U}-I_{s}\right\|+\left\|f_{N-1}\right\| o(h) \\
& =\left\|\widehat{V}^{N}-I_{s}\right\|+\frac{\left\|f_{n-1}\right\|}{N}(N o(h)) \rightarrow\left\|\exp \{P T\}-I_{s}\right\| .
\end{aligned}
$$

The last relationship follows from the fact that $\frac{\left\|f_{n-1}\right\|}{N}$ is bounded from part [i], whereas $N o(h)=\frac{o(h)}{h} \rightarrow 0$, as well as the fact that $\widehat{V}=I+h P+o(h)$. From the properties of the limit the proof is complete.

Acknowledgments. We are grateful to Jorge Moré for advice and assistance on limited-memory BFGS methods. We are also grateful to the anonymous referees for comments that have vastly improved the paper, particularly their suggestions to examine the effects of numerical errors (section 4) and the experimental setup in section 5.2.5.

\section{REFERENCES}

[1] A. Apte, D. Auroux, and M. Ramaswamy, Variational data assimilation for discrete Burgers equation, Electron. J. Differential Equations, 19 (2010), pp. 15-30.

[2] K. E. AtKinson, An Introduction to Numerical Analysis, John Wiley \& Sons, New York, 2008.

[3] D. P. Bertsekas, Dynamic Programming and Optimal Control, Athena Scientific, Belmont, MA, 1995.

[4] R. H. Byrd, J. Nocedal, and R. B. Schnabel, Representations of quasi-Newton matrices and their use in limited memory methods, Math. Programming, 63 (1994), pp. 129-156.

[5] P. Courtier, J. N. Thepaut, and A. Hollingsworth, A strategy for operational implementation of 4D-Var, using an incremental approach, Quart. J. Roy. Meteorological Soc., 120 (1994), pp. 1367-1387.

[6] R. Daley, Atmospheric Data Analysis, Cambridge University Press, Cambridge, UK, 1993.

[7] A. Geist and S. Dosanjh, IESP exascale challenge: Co-design of architectures and algorithms, Internat. J. High Performance Comput. Appl., 23 (2009), pp. 401-402.

[8] J. Glimm, S. Hou, Y. H. Lee, D. H. Sharp, and K. Ye, Sources of uncertainty and error in the simulation of flow in porous media, Comput. Appl. Math., 23 (2004), pp. 109-120.

[9] E. Kalnay, Atmospheric Modeling, Data Assimilation and Predictability, Cambridge University Press, Cambridge, UK, 2003.

[10] F. X. Le Dimet And O. Talagrand, Variational algorithms for analysis and assimilation of meteorological observations: Theoretical aspects, Tellus Ser. A, 38 (1986), pp. 97-110.

[11] J. M. Lewis, S. Lakshmivarahan, and S. K. Dhall, Dynamic Data Assimilation. A Least Squares Approach, Encyclopedia Math. Appl. 104, Cambridge University Press, Cambridge, UK, 2006.

[12] M. Lindskog, D. Dee, Y. Trémolet, E. Andersson, G. Radnóti, and M. Fisher, A weakconstraint four-dimensional variational analysis system in the stratosphere, Quart. J. Roy. Meteorological Soc., 135 (2009), pp. 695-706.

[13] M. J. Martin, M. J. Bell, AND N. K. Nichols, Estimation of systematic error in an equatorial ocean model using data assimilation, Internat. J. Numer. Methods Fluids, 40 (2002), pp. $435-444$.

[14] I. M. Navon, Practical and theoretical aspects of adjoint parameter estimation and identifiability in meteorology and oceanography, Dynamics of Atmospheres and Oceans, 27 (1998), pp. 55-79.

[15] J. Nocedal and S. J. Wright, Numerical Optimization, Springer-Verlag, New York, 1999.

[16] D. Orrell, L. Smith, J. Barkmeijer, And T. N. Palmer, Model error in weather forecasting, Nonlinear Processes Geophys., 8 (2001), pp. 357-371. 
[17] J. M. Ortega and W. C. Rheinboldt, Iterative Solution of Nonlinear Equations in Several Variables, Classics Appl. Math. 30, SIAM, Philadelphia, 2000.

[18] T. N. Palmer, G. J. Shutts, R. Hagedorn, F. J. Doblas-Reyes, T. Jung, and M. LeutBECHER, Representing model uncertainty in weather and climate prediction, Annu. Rev. Earth Planet. Sci., 33 (2005), pp. 163-193.

[19] G. W. Platzman, An exact integral of complete spectral equations for unsteady onedimensional flow, Tellus, 16 (1964), pp. 422-431.

[20] F. Rabier, H. Jarvinen, E. Klinker, J. F. Mahfouf, and A. Simmons, The ECMWF operational implementation of four-dimensional variational assimilation, I: Experimental results with simplified physics, Quart. J. Roy. Meteorological Soc., 126 (2000), pp. 11481170.

[21] L. R. RABiner, A tutorial on hidden Markov models and selected applications in speech recognition, Proc. IEEE, 77 (1989), pp. 257-286.

[22] L. Rabiner And B. JuAng, An introduction to hidden Markov models, IEEE ASSP Mag., 3 (1986), pp. 4-16.

[23] G. W. Stewart, Matrix Algorithms. Volume II: Eigensystems, SIAM, Philadelphia, 2001.

[24] Y. TrÉmolet, Accounting for an imperfect model in 4D-Var, Quart. J. Roy. Meteorological Soc., 132 (2006), pp. 2483-2504.

[25] Y. TrÉmolet, Model-error estimation in 4D-Var, Quart. J. Roy. Meteorological Soc., 133 (2007), pp. 1267-1280.

[26] F. Uboldi And M. KAMAChi, Time-space weak-constraint data assimilation for nonlinear models, Tellus Ser. A, 52 (2000), pp. 412-421.

[27] B. A. van de Rotten and S. M. V. Lunel, A Limited Memory Broyden Method to Solve High-Dimensional Systems of Nonlinear Equations, Tech. report 2003-06, Mathematical Institute, Leiden University, Leiden, The Netherlands, 2003. Available online at www.math.leidenuniv.nl/en/reports/997.

[28] M. Zupanski, D. Zupanski, T. Vukicevic, K. Eis, and T. V. HaAr, CIRA/CSU fourdimensional variational data assimilation system, Monthly Weather Rev., 133 (2005), pp. 829-843. 